\title{
Pathos der Funktion. Leonardos technische Zeichnungen
}

Eine späte, in Windsor Castle (Windsor, Royal Library $=$ W) aufbewahrte Federzeichnung Leonardo da Vincis verkehrt geläufige Vorstellungen der katastrophenträchtigen Beziehung zwischen Natur und Kultur (Abb. 1). Anders als auf seinen einige Jahre später entstandenen ,Sintflutzeichnungen“ ist es nicht der größte anzunehmende Unfall der Meteorologie, der die Hervorbringungen des Menschen vernichtet, sondern umgekehrt die Flut der Werkzeuge und Instrumente selbst, die als gefährlicher Hagelschlag auf die Erdoberfläche prasselt. Man erkennt Zangen, Hämmer, Nägel, Winkeleisen, Brillen, Zirkel, Rechen, Flaschen, Dudelsäcke, Leitern, Uhren, Teller, Scheiben usw., außerdem, in den Wolken, einen Löwen. Im Unterschied zur oberen Beischrift „Hier Adam und hier Eva“ -, die evtl. zu einer zweiten, später abgetrennten Zeichnung gehört haben könnte ${ }^{1}$, lässt die Bildunterschrift kaum Zweifel an der pessimistischen Bedeutung der Darstellung. „O miseria umana, di quante cose per danari ti fai servo.“ - „Oh Elend des Menschen, wie vieler Dinge Sklave bist Du doch wegen des Geldes.“ In Verbindung mit dem Wolkenlöwen wird aus der Skizze ein Prodigium (Abb. 2), das vielleicht auf die sprichwörtliche Technophilie der Florentiner - Stadt des Marzocco - anspielt oder eine Kryptosignatur Leone-Leonardos ${ }^{2}$ enthält. Für wahrscheinlicher halte ich jedoch, dass es sich bei dem Löwen um den 1513 gewählten Papst Leo X. de' Medici handelt, an den Leonardo große Hoffnungen knüpfte, die indes in Rom

1 Vgl. Kenneth Clark. The Drawings of Leonardo da Vinci in the Collection of $H$. M. The Queen at Windsor Castle. London, 1968, Bd. 1, sub num.

2 Vgl. Pierce Dominic Britton. „Lionizing Leonardo. A Physiognomic Conceit in Vasari's ,Vite““. Source 22.4 (2003), S. 10-15; Cecilio Paniagua. „Notes on a Drawing by Leonardo da Vinci“. International Review of Psychoanalysis 13 (1986), S. 445-452. 


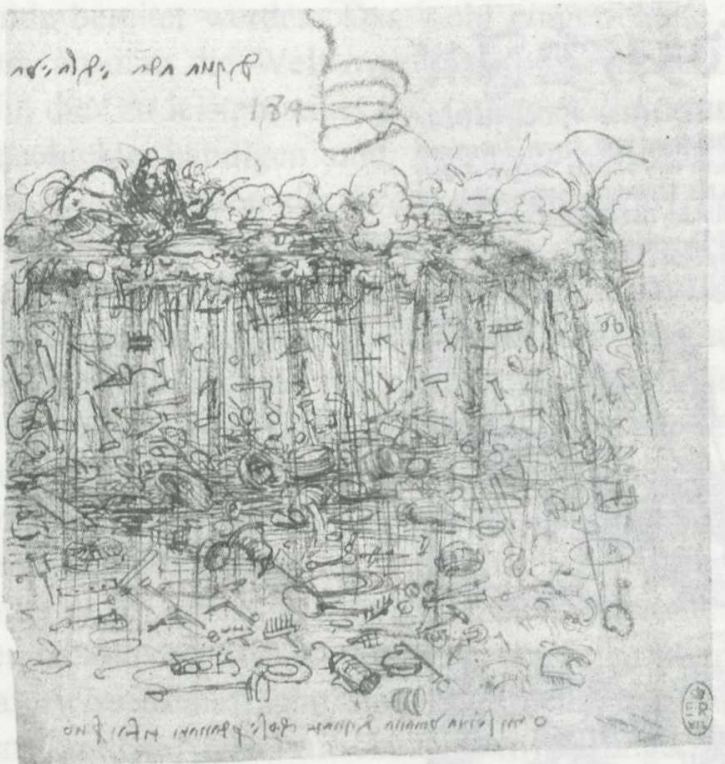

Abb. 1: Leonardo da Vinci. Sintflut der Werkzeuge. Feder und Kreide, ca. 1510-15. Windsor, Royal Library, Nr. 12698 recto.

enttäuscht wurden, was zu bitteren - und teilweise verschlüsselten Klagen Leonardos führte. Bekanntlich wurde Leonardo beim Papst wegen seiner anatomischen Forschungen denunziert. ${ }^{3}$ Es läge also nahe, das Blatt als Allegorie des Grundlagenforschers Leonardo gegen die utilitaristische Beschränkung der instrumentellen Sphäre ,im Zeichen Leos' zu deuten. Jedenfalls meint Leonardos antihumanistische Verbitterung unmissverständlich: Es ist die Habsucht, die zur unaufhörlichen, die gesamte Erdoberfläche bedeckenden Sintflut der Werkzeuge führt, Kehrseite und Motor des Entdecker- und Vermessungspathos, für das andere Texte unseres Meistertechniten stehen. ${ }^{4}$

3 Zu Leonardos Klage vgl. Codex Atlanticus, fol. 500. Vgl. auch Leonardos kryptische Notiz aus jener Zeit: „li medjci mi crearono e desstrussono" (Codex Atlanticus, fol. 429r; hierzu Carlo Pedretti. The Literary Works of Leonardo da Vinci. Commentary. London, 1977, Bd. 2, S. 313f., der einen Bezug zur Familie bezweifelt). Zur Gleichsetzung von Löwe und Leo X. und zur entsprechenden MediciIkonologie vgl. Suzanne B. Butters. The Triumph of Vulcan. Sculptor's Tools, Porphyry, and the Prince in Ducal Florence. Florenz, 1996, Bd. 1, S. 58f. Zum mechanischen Löwen, den Leonardo für die in Lyon ansässigen Florentiner anlässlich des Einzugs von François I. (12.07.1515) entwarf vgl. Pedretti (Anm. 3), S. 303.

$4 \mathrm{Zu}$ einer verwandten Darstellung Maarten van Heemskercks, auf welcher der Globus von Instrumenten und Werkzeugen bedeckt ist (1572). Zur Abb. vgl. Ilja M. 


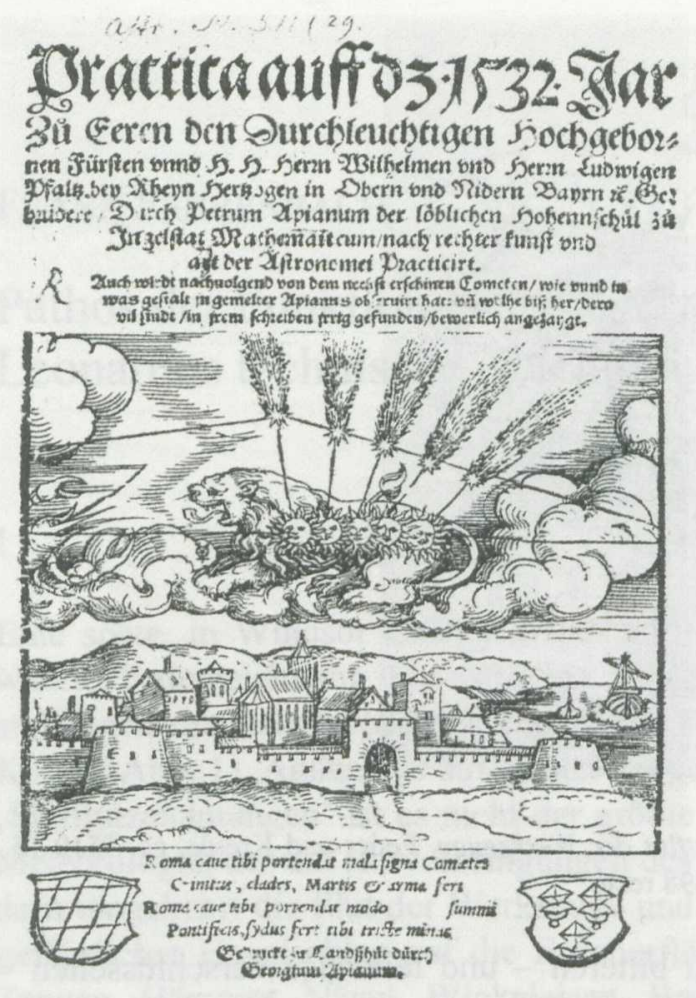

Abb. 2: Peter Apian. Practica für 1532 (Landshut, 1531).

Es ist evident, dass dasjenige, was vermisst, ordnet, begradigt, zurichtet selbst einer Ordnung bedarf. Die Bildgeschichte der Instrumente zeigt, dass ihnen auch noch als Trophäen der Wissenschaft eine geheime Abundanz und Widerspenstigkeit anhaftet. Unter der Herrschaft der während des Dreißigjährigen Krieges allegorisch als Landsknecht auftrumpfenden neuen Leitwissenschaft ${ }^{5}$ - der Mechanik - soll dem ein

Veldman u. Ger Luijten. Maarten van Heemskerck. (= The New Hollstein. Dutch \& Flemish Etchings, Engravings, and Woodcuts 1450-1700). Amsterdam, 1994, Bd. 2, Nr. 501/1. Vgl. Horst Bredekamp. „Der Mensch als ,zweiter Gott‘. Motive der Wiederkehr eines kunsttheoretischen Topos im Zeitalter der Bildsimulation“. Interface 1. Elektronische Medien und künstlerische Kreativität. Hg. v. Klaus Peter Dencker. Hamburg, 1992, S. 134-147.

5 Vgl. etwa den Stich „Mechanica und ihre Töchter und Söhne“ in Joseph Furttenbachs Mechanische[m] Reißladen. Augsburg, 1644. Vgl. dazu Jutta Bacher. „Das Theatrum machinarum. Eine Schaubühne zwischen Nutzen und Vergnügen“. Erkenntnis - Erfindung - Konstruktion. Studien zur Bildgeschichte von Naturwissenschaften und Technik vom 16. bis zum 19. Jahrhundert. Hg. v. Hans Holländer. 
Ende bereitet werden. Das wohl eingerichtete Labor ist nicht nur der Ort, an dem die Welt in logisch-numerische Ordnung gebracht wird. Um dies zu leisten, muss das Labor zuallererst die Fülle der Interventionsobjekte bändigen und instrumentelle Aufräumarbeit vollbringen. Die Klassifikation der Welt geht Hand in Hand mit der räumlichen Disposition der Instrumente. Wenn die Herrschaft über sie entgleitet, kehrt ein altes Chaos wieder, als Zeitalter post technologiam.

Leonardos Zeichnung, Menetekel der ,zweiten Natur', die durch die menschliche Technik realisiert wird ${ }^{6}$, enthält vielleicht auch die Palette eines Malers, aber nicht - das wäre kaum darstellbar - eines der kleinsten und gleichwohl raffiniertesten Instrumente der Weltaneignung: das Gerät des Zeichners. Erst der Feder verdankt aber Leonardos Blatt seine Existenz, eine nicht unerhebliche Differenz zum massenhaften Allerlei der gar nicht himmlischen Werkzeuge. Es ist genau diese Differenz, die den Stift an den Nullpunkt aller Instrumente heranrückt. Die naturwissenschaftliche Siegesgeschichte der Frühen Neuzeit wäre ohne jene kleinen, aber entscheidenden Sprünge zwischen mentalem und materiellem Entwurf, die der Zeichenstift garantiert, undenkbar gewesen. Das war natürlich schon den Pionieren dieses Instruments bewusst. Leonardo selbst spricht es klar aus ${ }^{7}$ : Das Zeichnen ist gerade mal ein Bewegen des Punktes; keine Handarbeit, sondern die kaum den Schatten einer körperlichen Spur hinterlassende kleine Geste einer „leichten Hand": la pittura è mentale. ${ }^{8}$ Dennoch hängt die menschliche Kultur buchstäblich am Tropf einer beinahe unausgedehnten Federspitze. „Der Anfang der Malerei ist der Punkt, dann folgt die Linie, das Dritte ist die Fläche. ${ }^{\text {"9 }}$ - Genau an dieser Stelle macht die Anregung der Herausgeber Sinn, eine Figur wie Leonardo in eine Tagung zur instrumentellen Kultur des 17. Jahrhunderts zu inserieren.

Für Leonardo wären ohne pittura die artes insgesamt unmöglich, weil ihre Kodierungen sichtbare Signifikanten benötigen. Malerei - und das heißt hier: Zeichnung, denn von der Farbe ist nicht die Rede - hat

Berlin, 2000, S. 514 sowie dies. „,Ingenium vires superat‘. Die Emanzipation der Mechanik und ihr Verhältnis zu Ars, Scientia und Philosophia“. Ebd., S. 519-555.

6 „Die Schwere, die Kraft, gemeinsam mit der materiellen Bewegung und der Stoß sind die vier akzidentiellen Potenzen, mittels derer die menschliche Gattung in ihren bewundernswürdigen und vielfältigen Handlungen in dieser Welt wie eine zweite Natur erscheint. Denn alle sichtbaren Werke der Sterblichen haben durch diese Potenzen ihr Sein und ihren Tod." (Leonardo, Codex Arundel, fol. 151v; ca.1495-97).

7 Vgl. Leonardo da Vinci. Manuskript E, fol. 34v.

8 Leonardo da Vinci. Libro di Pittura. Hg. v. Carlo Pedretti. Florenz, 1995, § 31c.

9 Ebd., $\$ 3$. 
nicht nur der Sprache die Schrift, der Arithmetik die Zahlen, der Geometrie die Figuren gegeben, sie lehrt auch Perspektiviker, Astronomen, Maschinenbauer und Ingenieure (,questa insegna alli prospettivi et astrologhi et alli machinatori et ingegneri“"). ${ }^{10}$ Die Zeichnung erweist sich so nicht nur als Erkenntnismedium, sondern als die Urerfindung schlechthin, aus der die anderen menschlichen Inventionen erst hervorgehen können. Damit wird übrigens eine Inserierung der Malerei ins Schema der artes liberales obsolet. Pittura nimmt einen Sonderstatus ein, der unter umgekehrten Vorzeichen nur noch mit der Theologie verglichen werden kann.

Es ist klar, dass sich hinter der Weichenstellung, die den sichtbaren Signifikanten keine dienende, sondern eine generative Funktion einräumt, grundlegende erkenntnistheoretische Vorentscheidungen verbergen, deren Fluchtlinie die spätmittelalterliche Karriere des aristotelischen phantasma darstellt. Per imaginem ad ideam: Das ergibt eine klare Sukzession, zumal wenn die inneren Bilder wasserdicht mit ihrem Entstehungsgrund, den Sinnen, verbunden sind. ${ }^{11}$ Dennoch wirken natürlich im Lob des Kleinen, Schnellen, nahezu Unausgedehnten ältere platonisierende Leitbilder fort. So bei Leon Battista Albertis und Leonardos Apologie des Auges und des Punktes, den der Zeichenstift, selbst ein nusquam, auf dem Blatt eindrückt und dabei, bewegt, eine Spur: die Linie zurücklässt. ${ }^{12}$ Das materielle Medium der Zeichnung verflüchtigt sich - dem spiritus gleich - zur Übergangsstelle zwischen Geist und Körperwelt. Wenig später lässt sich das gegen die Sinne selbst wenden und die Kunst zu einer Domäne des Verstandes machen, wie Georges Didi-Huberman gezeigt hat. Vasari schreibt:

Die Zeichnung [...] geht aus dem Intellekt hervor und schöpft aus vielen Dingen ein allgemeines Urteil, gleich einer Form oder Idee aller Dinge der Natur, die in ihren Maßen überaus regelmäßig ist. So kommt es, daß die Zeichnung nicht nur in den menschlichen und tierischen Körpern, sondern auch in den Pflanzen, Gebäuden, Skulpturen und Gemälden das Maßverhältnis des Ganzen in bezug auf die Teile sowie das Maßverhältnis der Teile untereinander und zum Ganzen erkennt. ${ }^{13}$

10 Ebd., § 23, vgl. ebd., § 31b. - Eine gute Zusammenstellung von Zeichengeräten bei Maja Hambly. Drawing Instruments. 1580-1980. London, 1988.

11 Vgl. Frank Fehrenbach. Licht und Wasser. Zur Dynamik naturphilosophischer Leitbilder im Werk Leonardo da Vincis. Tübingen, 1997, S. 17ff.

12 Vgl. Leon Battista Alberti. De pictura 1, 2: „Puncta quidem si continenter in ordine iungantur lineam extendent. Erit itaque apud nos linea signum cuius longitudo sane in partes dividi possit. Sed erit usque adeo latitudine tenuissima ut nusquam findi queat."

13 Giorgio Vasari. Leben der ausgezeichnetsten Maler, Bildhauer und Baumeister 
Dies ist das Geburtsdokument der ,Komposition", die zugleich vom belebten Körper abgeleitet wird, denn die wechselseitige proportionale Zweckmäßigkeit von Teilen und Ganzem ist bei Aristoteles formale Grundlage des Organismus. ${ }^{14}$

Wenig später, bei Federico Zuccari, wird dann die Verschmelzung von idea und disegno vollzogen, wobei der disegno auch noch die Linie seines dünnen Stiftes und den emanativen Punkt seiner Spitze eingebüßt hat: „Quasi eine andere Gottheit, eine andere naturierende Natur, in der die von der Kunst hervorgebrachten Dinge leben. [...] Inneres und äußeres Licht des Intellekts. [...] Nahrung und Leben jeder Wissenschaft und jeder Praxis. “ ${ }^{15}$ Aber die konstruktivistischen Fallen, die hier lauern, werden in der spezifischen Kultursituation des späteren 16. Jahrhunderts umgangen. Kunst bzw. Visualität mutiert stattdessen zur „Meta-Techne"16 einer experimentierfreudigen, aber doch primär kartographierenden und damit sammelnden Wissenschaftspraxis, die mithilfe der Zeichnung zunehmend den nichtnumerischen, „morphologischen“ Untersuchungsfeldern des 16. Jahrhunderts näher rückt. Das sind v.a. Biologie bzw. Medizin, Alchemie, Hydrologie und Geologie, die auf dem Baconischen Atlantis prominent residieren und noch von zahlreichen anticartesischen Wissenschaftlern des 17. und frühen 18. Jahrhunderts gemeinsam mit Perspektive und Optik erfolgreich bestellt werden. Pierre Gassendi findet für diesen Vorgang der zeichnerischen Weltaneignung 1658, gut baconisch, die passenden Worte: „Wir erforschen die Dinge der Natur in gleicher Weise, wie wir diejenigen Sachen untersuchen, die wir selbst geschaffen haben [...]. “17 Aber die Einheit

von Cimabue bis zum Jahre 1567. Übs. v. Ludwig Schorn u. Ernst Förster. Eingel. v. Julian Kliemann. Worms, 1988, Bd. 1, S. 63f. Zit. n. Georges Didi-Huberman. Vor einem Bild. Übs. v. Reinold Werner. München, 2000, S. 86.

14 Vgl. Aristoteles. De partibus animalium/Parts of animals. Griech./engl. Übs. v. A. L. Peck. Cambridge, Mass., 1983, 645b ff. Zur kontroversen Entstehungsgeschichte der frühneuzeitlichen ,Komposition“ vgl. Hans Körner. Auf der Suche nach der ,wahren Einheit'. Ganzheitsvorstellungen in der französischen Malerei und Kunstliteratur vom mittleren 17. bis zum mittleren 19. Jahrhundert. München, 1988; Thomas Puttfarken. The Discovery of Pictorial Composition. Theories of Visual Order in Painting 1400-1800. New Haven u. London, 2000; Frank Fehrenbach. „Komposition“. Metzler Lexikon Kunstwissenschaft. Ideen, Methoden, Begriffe. Hg. v. Ulrich Pfisterer. Stuttgart u. Weimar, 2003, S. 178-183.

15 Romano Alberti. Origini e progresso dell'Accademia del Disegno de' pittori, scultori e architetti di Roma. Pavia, 1604, S. 2060f. Zit. n. Didi-Huberman (Anm. 13), S. 91.

16 Vgl. Robert Williams. Art, Theory, and Culture in Sixteenth-Century Italy. From Techne to Metatechne. Cambridge, Mass., 1997.

17 Pierre Gassendi. Syntagma philosophicum. Lugduni, 1658. Zit. n. Hans Holländer. 
von Interpretation und Intervention, von Erkennen und Hervorbringen geht bereits, wie Wolfgang Krohn jüngst nachwies, auf die Bemühungen Albertis zurück, den wissenschaftlichen Status der Architektur neu zu begründen. ${ }^{18}$ Das bringt die Zeichnung als Medium des visuellen Experiments erst richtig ins Spiel. Ihr interventionistischer, konstruktiver Charakter bleibt jedoch stets an die visuelle Evidenz (und damit an den sensus communis der Adressaten) zurückgebunden; außerdem, im Falle des technischen Entwurfs, an seine funktionale Realisation.

Vor diesem bilder-, experimentier- und konstruktionsfreundlichen Hintergrund war die soziale Aufwertung des Zeichners geradezu unvermeidlich. Der dreizehnjährige Dürer stellte sich bereits 1484 auf der berühmten Silberstiftzeichnung der Albertina wie ein zweiter, lehrender Christusknabe dar, mit der weisenden Rechten des Zeichners, den aufmerksamen Blick fest auf ein unbekanntes Objekt gerichtet. ${ }^{19}$ Weniger noch als die Farben des Malers, der durch Leonardo den mit Marmorstaub verkrusteten, schwitzenden Bildhauer verhöhnte ${ }^{20}$, hinterließ das Zeichnen reinigungsintensive Spuren materieller Tätigkeit. So wiederholte sich quer durch die Malerzunft eine ältere Auseinandersetzung zwischen schmutziger und ,philosophischer" Tätigkeit. ${ }^{21}$ Auch farborientierte Malerfürsten taten gut daran, sich nicht von der Fraktion der (Florentiner) Intellektuellen vorführen zu lassen - und nahmen mitunter lieber demonstrativ den Zeichenstift als den Pinsel in die Hand; wie Tizian auf einem verschollenen Selbstporträt, das durch den Holz-

„Spielformen der Mathesis universalis“. Erkenntnis - Erfindung - Konstruktion. Studien zur Bildgeschichte von Naturwissenschaften und Technik vom 16. bis zum 19. Jahrhundert. Hg. v. dems. Berlin, 2000, S. 328.

18 Wolfgang Krohn. ,Technik, Kunst und Wissenschaft. Die Idee einer konstruktiven Naturwissenschaft des Schönen bei Leon Battista Alberti“. Leonardo da Vinci. Natur im Übergang. Beiträge zu Kunst, Wissenschaft und Technik. Hg. v. Frank Fehrenbach. München, 2002, S. 37-56.

19 Joseph Leo Koerner übersieht diese Analogie in der bislang umfangreichsten Studie zu den christomorphen Selbstbildnissen Dürers, obwohl er selbst auf die Parallele zum zeigenden Jesusknaben der Berliner Heiligen Familie von 1492-93 (Kupferstichkabinett, SMPK) verweist. Vgl. Joseph Leo Körner. The Moment of Self-Portraiture in German Renaissance Art. Chicago, 1993, S. 14 u. $42 \mathrm{ff}$.

20 Leonardo (Anm. 8), §36.

21 Zur Tradition des Kriteriums vgl. Claire J. Farago. Leonardo da Vinci's ,Paragone: A Critical Interpretation with a New Edition of the Text of the ,Codex Urbinas : Leiden u.a., 1992, S. 139ff.; ferner George Ovitt. The Restoration of Perfection. Labor and Technology in Medieval Culture. New Brunswick u. London, 1987. Besonders aufschlussreich: David Summers. The Judgment of Sense. Renaissance Naturalism and the Rise of Aesthetics. 2. Aufl. Cambridge, Mass., 1990, S. $259 \mathrm{ff}$. 


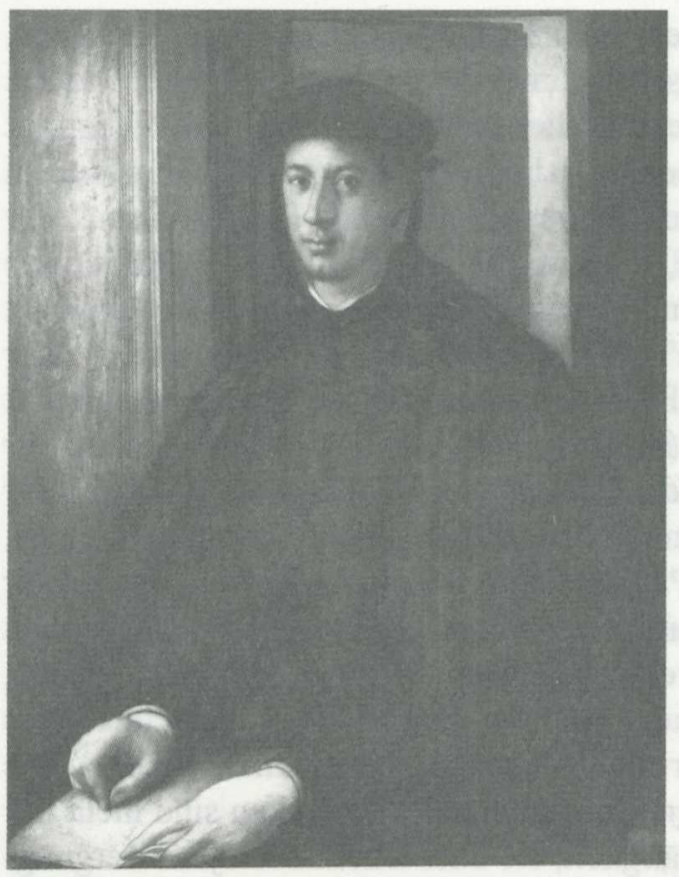

Abb. 3: Jacopo Pontormo. Alessandro de' Medici. Öl auf Holz, 1534. Philadelphia Museum of Art, Johnson Collection.

schnitt von Giovanni Britto aus dem Jahr 1550 überliefert ist. ${ }^{22} \mathrm{Zu}$ dieser Zeit hatte Baldassare Castiglione bereits in seinem verbindlichen Entwurf des noblen uomo universale das Zeichnen zur notwendigen Beschäftigung des Adels erklärt. ${ }^{23}$ Die Hinweise von Plinius und Alberti auf malende Patrizier und sogar Kaiser der Antike taten ihre Wirkung. ${ }^{24}$ Das spiegelt sich einzigartig schillernd in Jacopo Pontormos Porträt des wenig später ermordeten ersten Florentiner Herzogs, Alessandro de'Medici (Abb. 3). Politisch eine Gestalt, die schmutziger Arbeit nicht aus dem Weg ging und auch in Liebeshändeln durchaus mit

22 Joanna Woods-Marsden. Renaissance Self-Portraiture. The Visual Construction of Identity and the Social Status of the Artist. New Haven u. London, 1998, S. $228 \mathrm{f}$.

$23,[\mathrm{U}]$ n'altra cosa, la quale io [...] penso che dal nostro cortegiano per alcun modo non debba esser lasciata addietro: e questo è il saper disegnare ed aver cognizion dell'arte propria del dipingere." Baldassare Castiglione. Das Buch vom Hofmann. Übs. u. hg. v. Fritz Baumgart. München, 1986, Bd. I, S. XLIX.

24 Vgl. Gaius Plinius d. Ä. Historia naturalis/Naturkunde. Lat./deutsch. Übs. u. hg. v. Roderich König. München u. Darmstadt, 1973ff., Bd. XXXV, S. 20-22; Leon Battista Alberti. De pictura. Bd. II, 27. 
einem Hang zum Deftigen, erscheint er hier als zeichnender Feingeist, aber sein Maler hat sich selbst mit Alessandros Geliebter Taddea Malaspina vertauscht, die der fürstliche Bastard mit zartem Silberstift zeichnet; keine ewige Anbetung, aber eine ewige Nachahmung im Dienst der Verewigung eines schönen Äußeren. ${ }^{25}$ Der Chiasmus der Blicke macht aus der Dame, die das Bildnis empfing, die Porträtierte eines Porträts. Und die Trauerkleidung, die der galantuomo trägt, ist wohl nicht nur durch den Tod eines entfernteren Verwandten, aber wichtigsten Schutzpatrons, Papst Clemens VII. de'Medici, begründet (1534), sondern durch die implizite Abwesenheit der Geliebten. Ihr Bildnis rettet der Fürst, wie die Tochter eines Töpfers am Ursprung der Bildhauerei bzw. Zeichenkunst ${ }^{26}$, über die räumliche Trennung hinweg; in grauen Umrisslinien auf Papier, farbig in seinem Herzen - so wie er selbst als Abwesender in Farben vor seiner Geliebten erscheint. Schon vor dem drechselnden Souverän begegnen wir also dem zeichnenden, der seine Welt auf Papier bannt und der sich in die welterschließende und -unterwerfende geometrische Magie der Perspektive einübt. ${ }^{27}$ Die Klagen blieben nicht aus, denn instrumentelle Techniken sind nicht nur Analogien herrscherlicher Tätigkeit, sie entwickeln auch jene vergnügliche Eigendynamik, die jedem Dilettantismus eignet. Spätestens in Si-

25 Vgl. Philippe Costamagna. Pontormo. Mailand, 1994, Kat. Nr. 72.

26 Vgl. Plinius (Anm. 24), Bd. XXXV, S. 15 u. 151. - Bei Leonardo wird das erste Gemälde bezeichnenderweise durch einen Schattenwurf, den die Sonne verursachte, angeregt. Vgl. Leonardo da Vinci. Manuskript A, fol. 97v.

27 Vgl. dazu beispielsweise Jacopo de' Barbaris Brief an Friedrich den Weisen (1500/01): „Und ohne die ,Kenntnis‘ dieser ,sieben freien“ Künste kann kein glaubwürdiges Gemälde von den Malern hergestellt werden; es sei denn, sie beherrschen besagte Künste, zuerst die Geometrie, dann die Arithmetik, beides unabdingbare Voraussetzungen für die Proportionsbemessung. [...] Aber um diese Wissenschaften ,im Bild ' darzustellen, bedarf es erneut der Philosophie - und zwar der Ausführungen des Aristoteles über die Seele, wo er behandelt, wie die Bildeindrücke (specie) zum Auge gelangen und vermittels welcher Kenntnis der ,Licht-'Strahlen man die Gegenstände auf den leeren Bildtafeln anordnen kann. [...] Es fehlen nicht die Begabungen, aber es fehlen Muse und Adel! Denn zur Zeit Alexanders d. Gr. konnten die Kunst der Malerei nur Männer von adeligem Blut und Reichtum ausüben [...].“ Zit. n. Ulrich Pfisterer (Hg.). Die Kunstliteratur der italienischen Renaissance. Eine Geschichte in Quellen. Stuttgart, 2002, S. 268f. Zur Transformation der Perspektive im 16. Jahrhundert vgl. zusammenfassend Martin Kemp. The Science of Art. Optical Themes in Western Art from Brunelleschi to Seurat. New Haven u. London, 1990, S. 92. Zur Rolle des Dilettantismus: Wolfgang Kemp. ,... einen wahrhaft bildenden Zeichenunterricht überall einzuführen: Zeichnen und Zeichenunterricht der Laien 1500-1870. Ein Handbuch. Frankfurt a.M., 1979. Vgl. Klaus Maurice. Der drechselnde Souverän. Materialien zu einer fürstlichen Maschinenkunst. Zürich, 1985. 
tuationen, in denen die Existenz des Gemeinwesens auf dem Spiel steht, sollten die Fürsten darauf verzichten, ,das Pferd auf der Reitbahn herumzutreiben, zu schnitzeln, zu drechseln, zu malen, Alchimisterei und andere ihrem Stande ganz unangemessene Dinge zu treiben", wie der fürstlich Liegnitzische Leibarzt und Rat Caspar Dornau zu Beginn des Dreißigjährigen Krieges anmahnte. ${ }^{28}$ Die Reihenfolge seiner Aufzählung macht Malerei zu einer nahen Verwandten einer spekulativen und magischen Kunst. Man sollte das Pathos dieser nachahmenden Weltneuschöpfung nicht unterschätzen. In Enea Vicos Darstellung der Accademia Baccio Bandinellis (um 1550) sind die mehr oder minder schlaftrunkenen Schüler damit beschäftigt, in tiefer Nacht, bei Feuerschein und Kerzenlicht sowohl antike Statuenfragmente als auch Skelettteile zu zeichnen - eine alchemistische Laborsituation; auch dies im Dienst der Neubelebung zerstückelter steinerner und fleischloser Körper. $^{29}$

II

Schon die bloße Menge der erhaltenen technischen Zeichnungen Leonardos (und damit nur ein kleiner Bruchteil des ursprünglichen Bestandes) ist Furcht erregend: ca. sechstausend Einzelblätter und Manuskriptseiten, wohl der umfangreichste technikgeschichtliche Nachlass der Renaissance. Vieles blieb erhalten (Vasari spricht in diesem Zusammenhang bezeichnenderweise von Reliquien ${ }^{30}$ ), weil es am Ingenium des künstlerischen Personalstils partizipierte. Was wäre aus Leonardos technischen Skizzen geworden, wenn seine Umgebung nicht doch und wohl ganz in Leonardos Sinn - in ihm vor allem den Maler des Mailänder Abendmahls und der unvollendeten Schlacht von Anghiari bewundert hätte? Der Löwenanteil dieser Zeichnungen entfällt auf vier Themenbereiche: Hydraulik, Militärtechnik, Flugmaschinen und zuletzt die zahllosen Blätter, auf denen sich Leonardo mit grundlegenden mechanischen Problemen und theoretischer Mechanik beschäftigt. Schon die Menge zeigt: Dieser Mensch legte den Zeichenstift wohl buchstäb-

28 Zit. n. Maurice (Anm. 27), S. 23.

29 Zum Stich und zu Bandinellis Akademie zuletzt Leonard Barkan. Unearthing the Past. Archeology and Aesthetics in the Making of Renaissance Culture. New Haven u. London, 1999, S. $289 \mathrm{ff}$,, dem allerdings die angesprochene Dialektik (Fragment und Ganzheit, tot und lebendig, dunkel und hell) entgeht.

30 Vgl. Giorgio Vasari. Le Vite de' più eccellenti pittori, scultori e architettori. Hg. v. Rosanna Bettarini u. Paola Barocchi. Florenz, 1987, Bd. 4, S. 28. 
lich kaum aus der Hand; er muss ununterbrochen gezeichnet und ebenso wichtig - eigene ältere Zeichnungen betrachtet haben. Die Spannweite der graphischen Dokumente ist groß und lässt sich idealtypisch in ein Dreieck einschreiben, das durch Protokoll, phantastischen Entwurf und Diagramm begrenzt ist. Die Zeichnungen stehen im Dienst der Verdeutlichung und gehören damit von vornherein einem rhetorischen Ambiente an, das sich die energeia des Visuellen zu Eigen macht. Technikgeschichtlich übernimmt bei Leonardo die Zeichnung erstmals in der gemischten Gattung der illustrierten Traktate den argumentativen Primat. ${ }^{31}$

Das technische Ingenium wird durch Leonardo erstmals auch sozial freigesetzt. Seine Position ist nicht an bestimmte Aufträge und Aufgaben gebunden. Als Ratgeber und Erfinder bewegt er sich - in Mailand, Florenz, Rom, zuletzt Amboise und beinahe auch einmal am Bosporus residierend - praktisch frei in und zwischen den jeweiligen Territorien; sein Einkommen als Genie der Maschinen ist häufig unabhängig von spezifischen Aufgaben. ${ }^{32}$ Leonardos singuläre Freiheit erfordert aber ein hohes Maß an flankierender Rhetorik. Von seiner außergewöhnlichen Eloquenz, die der graphischen wohl kaum nachstand, berichten unterschiedliche Quellen. ${ }^{33}$ Schon der berühmte, in einer Abschrift erhaltene Bewerbungsbrief ${ }^{34}$ an den damals mächtigsten Fürsten Italiens, Lodovico Sforza, bezeugt den Wagemut des Dreißigjährigen, denn die Versprechungen, die er macht, und ihr Duktus folgen einem mittelalterlichen Propheten der technischen Utopie, dem Franziskaner Roger Bacon, den Leonardo später ausführlich rezipiert. Unter den secreti miei, die Leonardo in Aussicht stellt, fungieren prominent Pontonbrücken, Kriegsschiffe, gepanzerte Wagen, Minentechnik und Tauchinstrumente: genau diejenigen Bewegungstechnologien auf und unter Erde und Wasser, von denen Roger Bacon zweihundert Jahre früher geschwärmt hatte und die, daran hat Horst Bredekamp erinnert, in einer ersehnten Zukunft zugleich die technologischen Wunder des Altertums

31 Als zeitgenössische Parallele wäre Francesco di Giorgio zu nennen. Vgl. dazu Paolo Galluzzi (Hg.). Prima di Leonardo. Cultura delle macchine a Siena nel Rinascimento. Mailand, 1991 (dort auch zahlreiche Beispiele unbeschrifteter technischer Illustrationen, beispielsweise im Ambiente von Mariano di Iacopo, gen. Taccola). Vgl. auch Herbert Maschat. Leonardo da Vinci und die Technik der Renaissance. München, 1989.

32 Vgl. Paolo Galluzzi. Gli ingegneri del Rinascimento da Brunelleschi a Leonardo da Vinci. Florenz, 2001, S. $47 \mathrm{ff}$.

33 ,[...] e con le parole volgeva al sì e al no ogni indurata intenzione." Vasari (Anm. 30), S. 37.

34 Leonardo da Vinci. Codex Atlanticus, fol. 1082. 


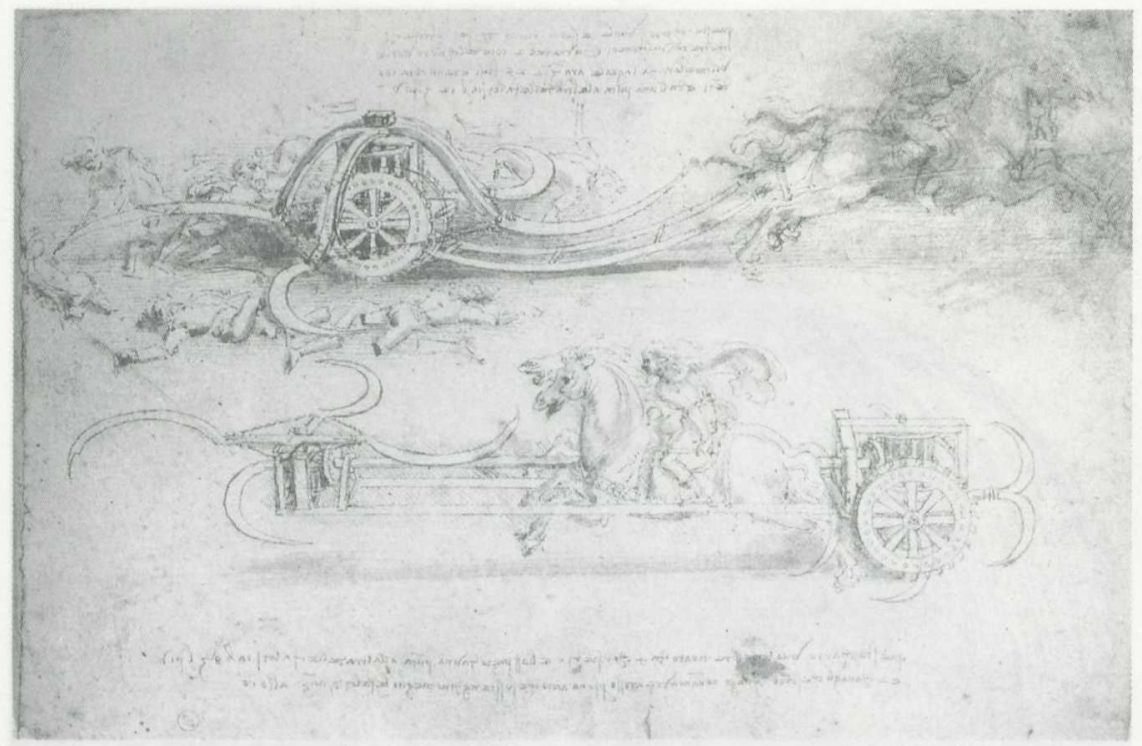

Abb. 4: Leonardo da Vinci. Sichelwagen. Feder und Bister, um 1490. Turin, Biblioteca Reale, Nr. 15583.

„erneuern“ sollten. ${ }^{35}$ In dieses antikisierende Umfeld gehören frühe Projekte wie der Sichelwagen (Abb. 4), bei dem Leonardo allerdings zu bedenken gibt, dass er wahrscheinlich bei den eigenen Leuten genauso viel Unheil anrichten wird wie bei den Feinden.

Das Ansehen, das Leonardo als Techniker genoss, die Bereitschaft auch skeptischer Regierungen wie derjenigen Venedigs oder der Florentiner Republik, gewaltige Arbeiten voranzutreiben (um sie dann scheitern zu sehen), die Fähigkeiten Leonardos, pragmatische Kontexte zu poetisieren und zu rhetorisieren, muss vor allem mit seinen zeichnerischen Kapazitäten verbunden werden. Ich möchte ihre Spezifika stichwortartig als Perspektivierung, Geometrisierung und - herausragend - Prozessualisierung bezeichnen.

Eine weitere, in Windsor aufbewahrte Zeichnung von ca. 1503/04 (Abb. 5), wohl im Umfeld der Bedrohung von Florenz durch Cesare Borgia anzusiedeln, spiegelt alle drei Kriterien in nuce. Dargestellt ist eine raffinierte Verteidigungstechnik, die es ermöglichen soll, dem Feind, der schon die äußeren Mauern einer Befestigungsanlage einge-

35 Vgl. Domenico Laurenza. „Leonardo. Le macchine volanti“. Le macchine del Rinascimento. Hg. v. Giovanni Morello. Rom, 2000, S. 145-187; Horst Bredekamp. Antikensehnsucht und Maschinenglauben. Die Geschichte der Kunstkammer und die Zukunft der Kunstgeschichte. Berlin, 1993. 


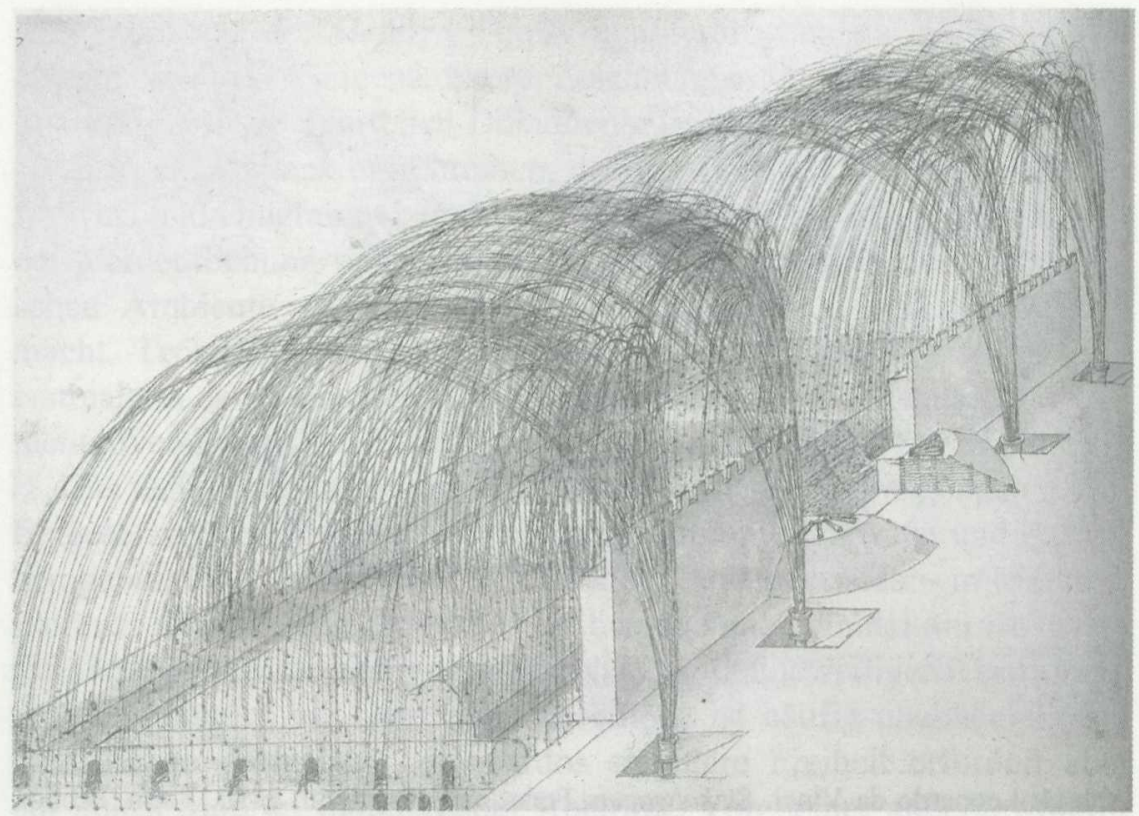

Abb. 5: Leonardo da Vinci. Militärtechnischer Entwurf. Feder, um 1503/04. Windsor, Royal Library, Nr. 12275.

nommen hat, durch ein Streubombardement zuvor unterirdisch verborgener Kanonen in den Rücken zu fallen. Leonardo stellt dies als aktuellen Vorgang dar und zeichnet zur Verdeutlichung die halbkreisförmigen Geschossbahnen ein, die sich in der makellosen Schönheit von Wasserspielen präsentieren. Der graphische Aufwand der verkürzten, elliptischen Kurven ist erheblich. Leonardo legte großen Wert darauf, jedes der am Boden liegenden Geschosse in exakt identischem Abstand zum nächsten Projektil darzustellen. Das signalisiert: Die Verteidigungstechnik partizipiert an der geometrischen Perfektion des perspektivischen Raumrasters. Der Vorgang findet ohne das anekdotische Beiwerk von angreifenden und gefallenen Soldaten auf vollständig planem, bereinigtem und gleichmäßig beleuchtetem Terrain statt. Der Betrachter wohnt einem idealen Prozess, einem programmierten Ablauf bei. Die Verteidigungsanlage ist, der besseren Einsicht zuliebe, durch einen sauberen Schnitt fragmentiert. Weder die Perspektive des Angreifers noch die der Eingeschlossenen wurde gewählt, sondern jene ideale, über den Mauern und jenseits der Reichweite der Bomben schwebende Schrägsicht, die später bezeichnenderweise zur so genannten Militärperspektive wurde. Diese Sicht kommt Befehlshabern auf imaginären Feldherrenhügeln zu. Der Betrachter von Leonardos Zeichnung, die die 


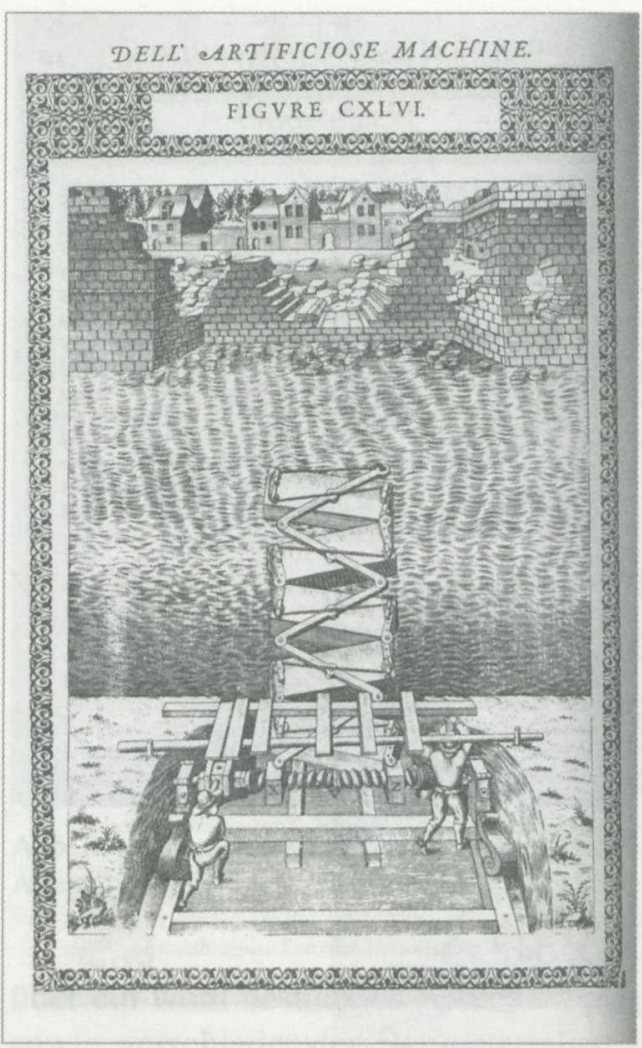

Abb. 6: Agostino Ramelli. Diverse et artificiose machine (Paris, 1588, fig. CXLVI).

Ästhetik des Videokrieges vorwegnimmt, versteht die Botschaft, die da lautet: Du, der Du mich betrachtest, kannst nur ein Stratege sein; Du hast die vollständige Durchsicht (perspectiva). - Die militärische Rhetorisierung der Perspektive, genauer: die Gleichsetzung von Durchsicht und Durchschlagskraft blieb nicht folgenlos, wie eine Illustration aus Agostino Ramellis Diverse et artificiose machine von 1588 belegen mag (Abb. 6).

Nur wer - wie Leonardo - Perspektive und die Anatomie des bewegten Körpers gleichermaßen zeichnerisch beherrscht, ist in der Lage, diesen persuasiven Ansatz fortzuspinnen und das Privilegium des Betrachters auf die Spitze zu treiben. Seine Darstellung einer Geschützgießerei (Abb. 7) entstand m.E. in den 1490er Jahren in Mailand, damals das militärtechnische Zentrum Europas. Die enorme Komplexität der Zeichnung darf nicht darüber hinwegtäuschen, dass einige Vorgänge recht unklar sind, insbesondere die äußerst kraftintensiven Tätigkeiten der Arbeiter im rechten Bildteil. Hauptthema ist das Aufsetzen 


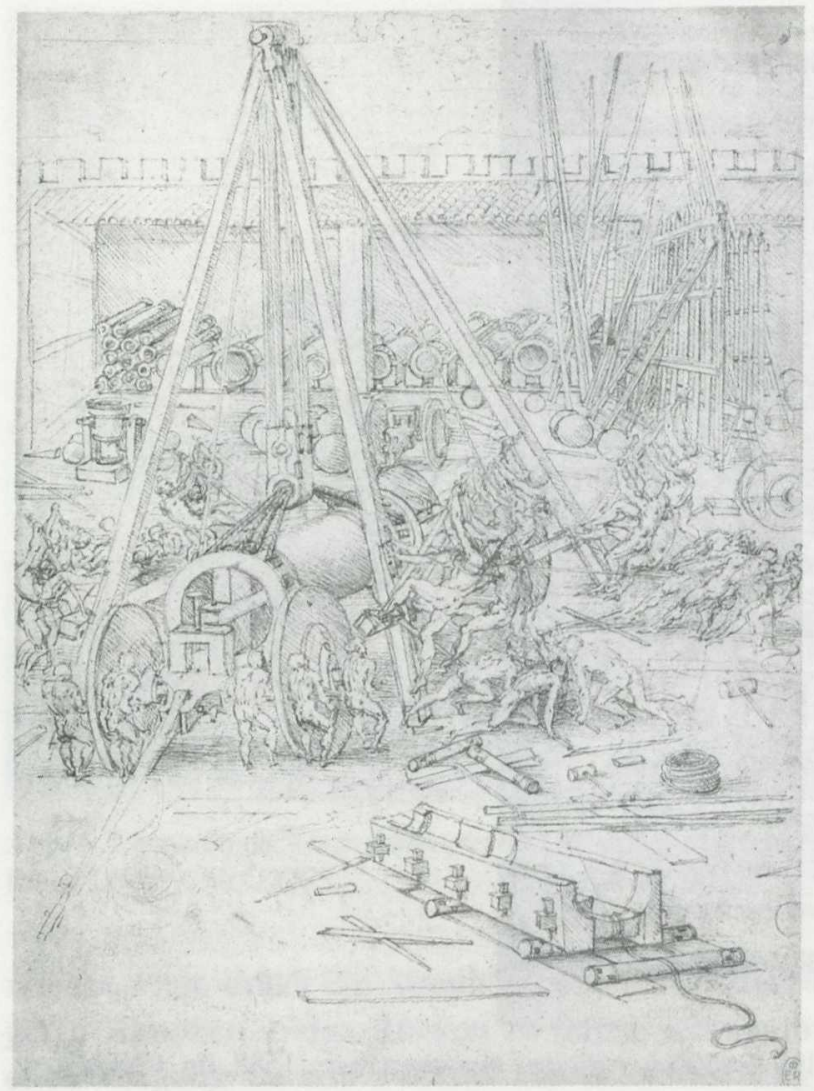

Abb. 7: Leonardo da Vinci. Geschützgießerei. Feder, um 1495. Windsor, Royal Library, Nr. 12647.

einer neu gegossenen gigantischen Kanone auf den Geschützwagen. Zahlreiche nackte Arbeiter sind mit unterschiedlichen, aber synchronisierten Verrichtungen beschäftigt, die den äußersten körperlichen Einsatz fordern. Die Zeichnung ist nicht nur darin rhetorisch, dass sie den fürs Ganze blinden, einzelnen Teams den Überblick des Betrachters gegenüberstellt; sie ist dies in besonderem Maße dadurch, dass sie den Arbeitsfuror ohne koordinierenden Vorarbeiter, also als automatisierten Arbeitsablauf direkt dem Betrachter präsentiert. $E r$ ist es, der zum idealen Aufseher bzw. Programmierer des Geschehens mutiert. Und nicht nur das: $E r$ ist es auch, der allein den vollständigen Überblick über die Fertigung von der Gussform im Vordergrund und ihrem Transport auf Rollen über den Einsatz der Flaschenzüge am Hebegerüst bis zur angedeuteten späteren Bestückung der Kanone mit Kugeln (im Hintergrund) behält und nebenbei auch noch als ordnender Aufseher 


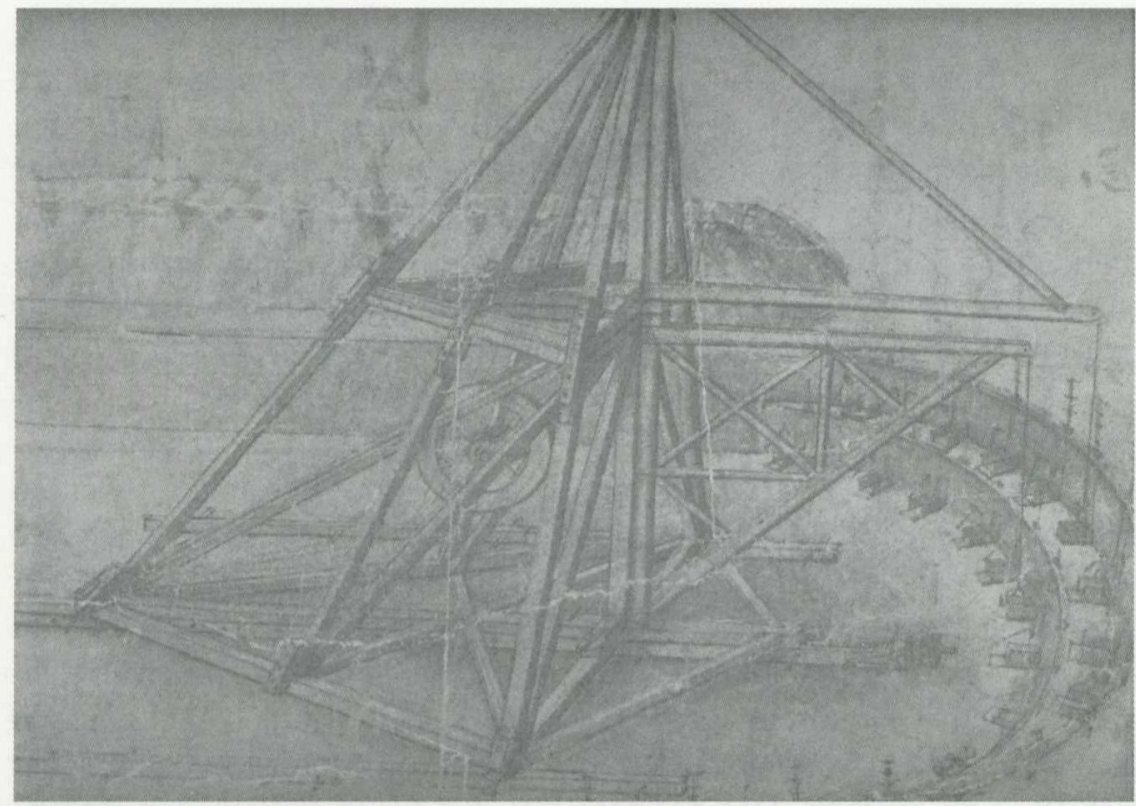

Abb. 8: Leonardo da Vinci. Entwurf eines Baggers. Feder und Bister, um 1500. Codex Atlanticus, fol. 4 recto. Mailand, Biblioteca Ambrosiana.

über ein wohl bestücktes Arsenal weiterer (überdachter) Kanonenrohre sowie verschiedenster Baumaterialien ins Spiel kommt. Während die hardware: alle Waffen, Gebäude, Werkzeuge klar umrissen und perspektivräumlich definiert ist, fungieren die unbekleideten Arbeiter als ephemere Kollektive, Gliederapparate, als vorübergehende Verkörperungen von Zug- und Schubkräften, deren Umrisslinien eigentümlich permeabel bleiben.

Die Koppelung von perspektivischem Ordnungssystem und Skizzenstatus des Dynamischen und Organischen ist es, die einen weiteren Gesichtspunkt ankündigt: das kalkulierte Nonfinito der technischen Zeichnung, die Rhetorik des Entwurfs. Leonardo war auch darin Pionier. Auch wenn es viele Zeichnungen wie diejenige des großen Baggers zur Ausschachtung von Flussbetten (Abb. 8) gegeben haben muss - Blätter, durch deren Vollendungsgrad die Handschrift des Zeichners beinahe verschwindet -, wird die Mehrzahl der Entwürfe, denen Leonardo seinen Ruhm verdankte, doch noch weitgehende Spuren ihrer Entstehung an sich getragen haben. Nicht nur, weil dies die Mehrzahl der erhaltenen technischen Zeichnungen nahe legt. Auch der künstlerische Werkprozess war bei Leonardo bekanntlich durch eine Aversion gegenüber der Vollendung und eine Bevorzugung erprobender, offener 
Verfahren „bis zuletzt“ geprägt, mit den bekannten desaströsen Folgen für die großen Freskenaufträge. ${ }^{36}$ Es muss geradezu ein wesentlicher Bestandteil der technischen Rhetorik Leonardos gewesen sein, den Adressaten Einblick in den Entwurfsprozess zu gewähren. Der Sekretär des Kardinals von Aragon, der Leonardo 1517 in Clos Lucé besuchte, berichtet, dass Leonardo stolz auf die lange Reihe seiner Manuskripte verwiesen habe - und wir wissen, wie , chaotisch ${ }^{6}$ die meisten dieser Manuskripte angelegt sind. ${ }^{37}$ Ich vermute, dass der , ingeniöse ${ }^{6}$ Zustand der technischen Projekte wesentlicher Teil des persuasiven Eros Leonardos gewesen war. 1504 unterstützte Leonardo das Vorhaben der Florentiner Republik - ein Lieblingsprojekt seines Freundes Niccolò Machiavelli - den Lauf des Arno vor Pisa so zu ändern, dass der alte Erbfeind vom Wasser abgeschnitten worden wäre. ${ }^{38}$ Die tatsächlich begonnenen Arbeiten an der Großbaustelle - über 2000 Arbeiter waren im Einsatz - mussten nach kurzer Zeit angesichts der nicht zu bewältigenden Aufgabe eingestellt werden. In dieser Zeit wurde von Leonardo auch das Projekt vorangetrieben, den Arno von Florenz bis zum Meer schiffbar zu machen. Das gesamte Val di Chiana südlich von Arezzo sollte als regulierendes Staubecken unter Wasser gesetzt und die Stromschnellen vor Empoli durch die weiträumige Verlegung des Flussbetts nach Norden umgangen werden. Teil des Planes war auch ein Kanaltunnel bei Serravalle (vor Lucca), durch den zuletzt das alte Flussbett wieder erreicht worden wäre. ${ }^{39}$ Das Vertrauen Leonardos in unbegrenzte Krafterzeugung war damals noch ungebrochen, auch wenn die Entwicklung der Flugmaschine bereits zur Vorsicht mahnte. ${ }^{40}$ Noch um

36 Zum Frühwerk vgl. Michael Wiemers. Bildform und Werkgenese. Studien zur zeichnerischen Bildvorbereitung in der italienischen Malerei zwischen 1450 und 1490. München u. Berlin, 1996, S. $265 \mathrm{ff}$.

37 Nach dem Besuch bei Leonardo in Cloux (10.10.1517) notierte Antonio de' Beatis: „Ha anche composto de la natura de l'acqua, de diverse machine et altre cose [man beachte die Reihenfolge!], secondo ha riferito lui, infinità di volumi et tutti in lingua volgare." Antonio de Beatis. Die Reise des Kardinals Luigi d'Aragona durch Deutschland, die Niederlande, Frankreich und Oberitalien 1517 bis 1518. Hg. v. Ludwig von Pastor. Freiburg, 1905, S. 143; dazu Carlo Pedretti. Leonardo da Vinci on Painting. A Lost Book (Libro A). Berkeley u. Los Angeles, 1964, S. 109 u. ders. (Anm. 3). Bd. 2, S. 140.

38 Vgl. dazu Nicolai Rubinstein. „Machiavelli and the Decoration of the Hall of the Great Council in the Palazzo Vecchio“. Musagetes. Festschrift für Wolfram Prinz. Hg. v. Ronald G. Kecks. Berlin, 1991, S. 275-285.

39 Vgl. Ladislao Reti. „Leonardo the Technologist. The Problem of the Prime Mover“. Ders. u. Bern Dibner. Leonardo da Vinci, Technologist. Norwalk, 1969, S. 90.

40 Vgl. Frank Fehrenbach. „Hier stehe ich, aber mein Auge durcheilt die Räume. Die 


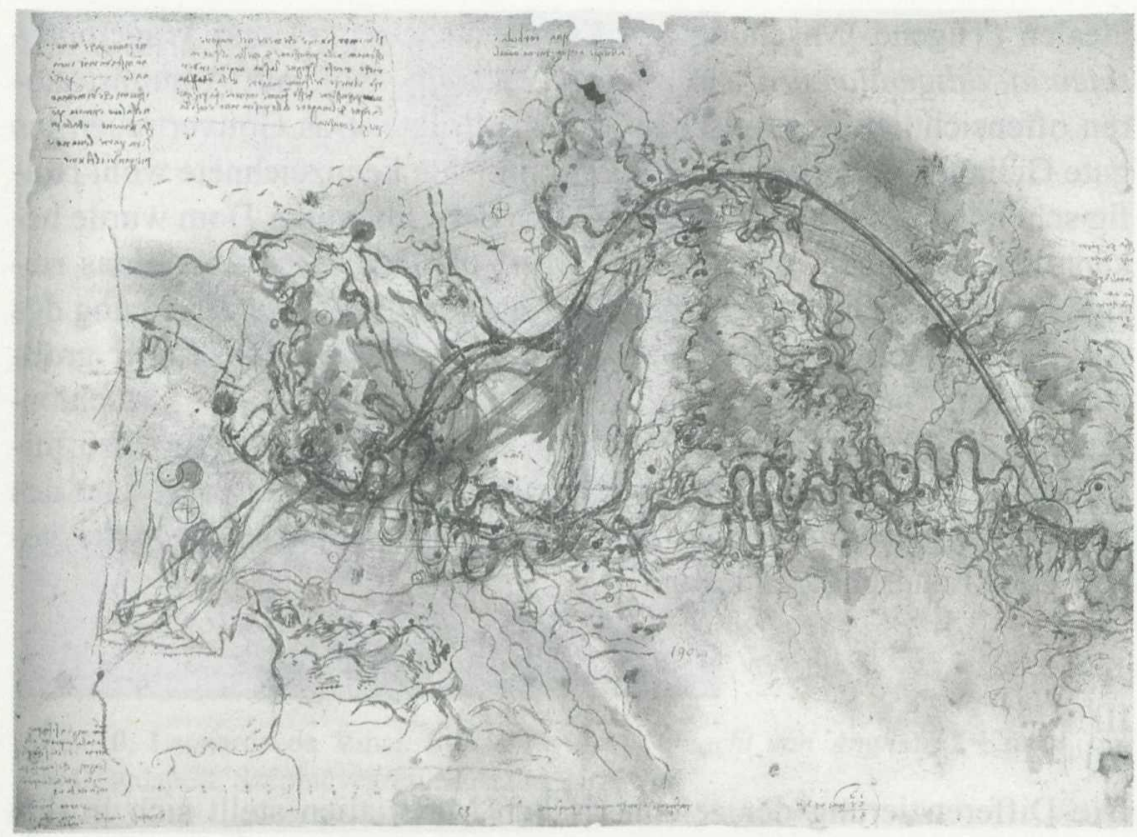

Abb. 9: Leonardo da Vinci. Karte des Arno. Mischtechnik, 1503/4. Windsor, Royal Library, Nr. 12279.

1495 spekulierte Leonardo darüber, mittels einer hypothetischen Kanone die Erde aus ihrem Zentrum im Weltall zu schießen - ein technoider Wachtraum, der in signifikanter Weise eine Brücke zwischen Archimedes, Schießpulver und Nikolaus Kopernikus baut. ${ }^{41}$

Die ebenfalls in Windsor befindliche große Karte von 1503/04 (Abb. 9) ist ein höchst komplexes Elaborat aus unterschiedlichen Materialien (Feder mit brauner Tinte, schwarze Kreide, Bisterlavierungen), die in kühnem Schwung den neuen Verlauf des Arno anzeigt; in ihrer Mischung aus geographischer Akribie (man beachte die Nebenflüsse des Arno!) und ,unreinen', alternativen Kanallinien, die dennoch mit großer Entschiedenheit gesetzt wurden, ein sprechendes Beispiel jener

Vogelstudien und Flugversuche von Leonardo da Vinci“. Frankfurter Allgemeine Zeitung (27.4.2002), S. 47.

41 Vgl. Leonardo da Vinci. Codex Madrid I, unnum. erstes Vorsatzblatt recto: „Se possibile fussi fare una bonbarda, che 'l mondo fussi sua ballotta, e che sicome una bonbarda gitta una balotta d'un braccio 3 miglia, che si pò misurare il tal corso 9000 braccia, cioè 9 mila ballotte. Noi possiamo adunque dire, che tal bonbarda gitterebbe il nostro mondo novemila volte la grandeza del diamitro d'esso mondo distante da ssè. [Sare]bono a settemila miglia per mondo, sarebono 63 migliara di miglia." 
idealen (Tugend-)Mischung aus Know-how, Sorgfalt und Wagemut scientia, diligentia/cura und potestas audendi -, die auf Administratoren offensichtlich hypnotischen Zwang ausübte. Das Gottvertrauen ins gute Gelingen unvorhersehbarer Großprojekte kennzeichnete wohl häufig schon damals die Regierungen; auch der Florentiner Dom wurde bekanntlich begonnen, ohne dass Klarheit darüber bestand, wie das riesige Kuppelrund jemals geschlossen werden sollte. ${ }^{42}$ Die Zeichnung des Arno gibt Einblick in den brodelnd-ingeniösen Urgrund solcher großtechnischer Entscheidungsprozesse; man sieht förmlich die Entschlossenheit, die Leonardo befeuerte, in großer Geste linkshändig, also mimetisch von rechts nach links, mit nassem Pinsel den neuen Verlauf des Flusses - vielleicht vor den Augen seines Publikums - über die gezeichnete Landschaft zu legen.

\section{III}

Die Differenzierung der zeichnerischen Materialien stellt sich in den Dienst eines erweiterten mimetischen Ansatzes. Leonardo operierte vor 1500 vorwiegend mit Metallstiften (die nicht korrigiert werden können) und, mehr noch, mit Federn verschiedener Stärke - wobei die Feder als gemeinsames Medium die behauptete Priorität der Zeichnung vor den sprachlichen Signifikanten besonders eindrucksvoll vor Augen stellt: Auch die Schrift ist eine Form der Zeichnung. ${ }^{43}$ Nach 1500 bringt Leonardo die unterschiedlichsten Medien zum Einsatz: Rötel, Schwarze Kreide, Pastell, Kohle und Aquarell. ${ }^{44}$ Den unterschiedlichen Medien liegen jeweils unterschiedliche Wirkungsabsichten zugrunde, die mit den Charakteristika der dargestellten Objekte verbunden sind. Schon zuvor lässt sich bei Leonardo eine sorgfältige mediale Stufung der Zeichnung beobachten, bei der das Blatt häufig zuerst mit dem trockenen Stilus, also durch farblose Prägelinien geisterhaft figuriert wird. Dies ist auch heute nur bei aufmerksamer Betrachtung in schrägem Licht sichtbar und lässt sich beim besten Willen nicht reproduzieren: Was wir in immer schwergewichtigeren Publikationen in Händen halten, ist nur die ,Außenseite ${ }^{6}$ eines Euvre, das ins Imponderable

42 Zum technikgeschichtlichen Aspekt der Unternehmung vgl. Daniela Lamberini. „All'ombra della cupola: tradizione e innovazione nei cantieri fiorentini quattro e cinquecenteschi“. Annali di architettura 10/11 (1998/99), S. 276-287.

43 Vgl. Leonardo (Anm. 8), § 23.

44 Vgl. dazu Francis Ames-Lewis. La matita nera nella pratica di disegno di Leonardo da Vinci (= Lettura Vinciana Bd. 41). Florenz, 2002. 


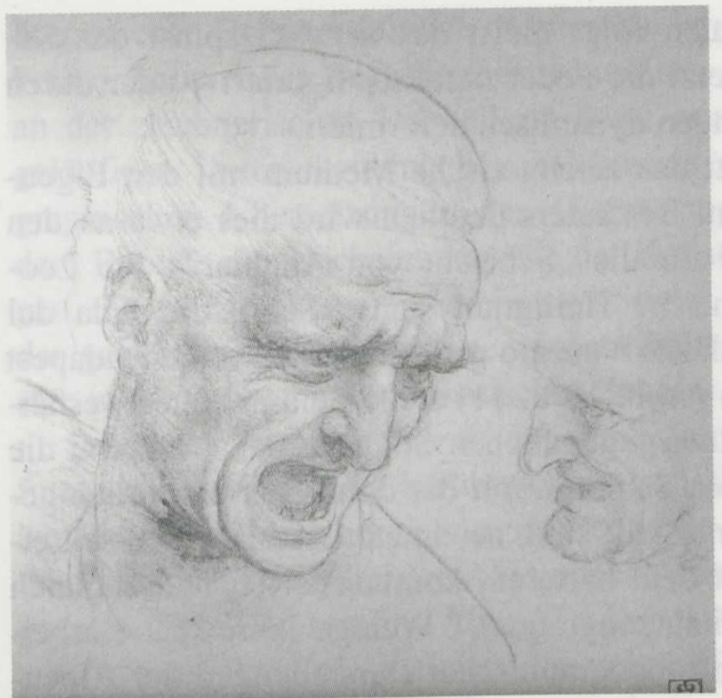

Abb. 10: Leonardo da Vinci. Studie für die „Schlacht von Anghiari“. Kreide, um 1504. Budapest, Szépmüvészeti Múzeum, Nr. 1774.

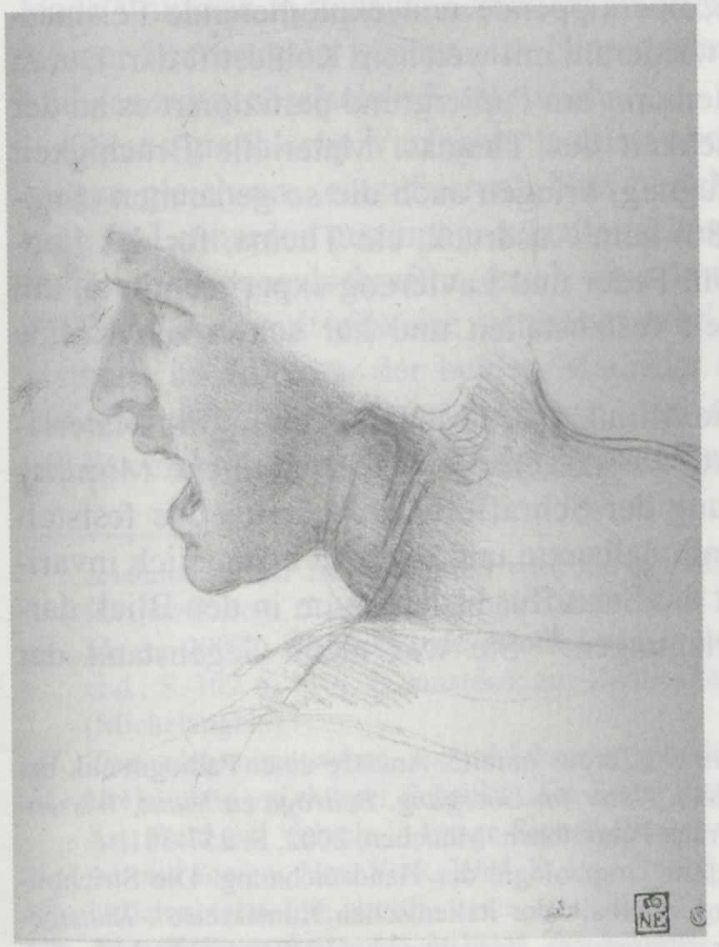

Abb. 11: Leonardo da Vinci. Studie für die „Schlacht von Anghiari“". Rötel, um 1504. Budapest, Szépmüvészeti Múzeum, Nr. 1775. 
spielt. Auf die Geisterlinien folgt meist das zarte Gespinst der Silberstiftzeichnung, bis zuletzt die Feder definitiv figuriert - oder durch immer neue Überzeichnungen dynamisch deformiert.

Nach 1500 verschmilzt das zeichnerische Medium mit den Eigenschaften des Dargestellten. Besonders deutlich wird dies etwa an den großen Ausdrucksstudien für die "Schlacht von Anghiari“, die Leonardo für das republikanische Heiligtum seiner Stadt, die Sala del Maggior Consiglio des Palazzo Vecchio malen sollte. Zwei in Budapest aufbewahrte Zeichnungen (Abb. 10 u. 11) stellen einmal ältere, erfahrene Haudegen, einmal einen jugendlichen Soldaten dar. Während die wutverzerrten, faltenreichen Senioren mit der dunkleren und brüchigeren Schwarzen Kreide dargestellt sind, zeichnete Leonardo den schreienden jungen Krieger mit dem härteren, konturscharfen und zugleich ,blutfarbenen' Rötelstift (ital. sanguigna). ${ }^{45}$ Wenige Jahre später arbeitet Leonardo an einer Serie topographischer Darstellungen der Alpenkette (W 12414), für deren festgefügten Bestand wiederum Rötel - auf rotgefärbtem Papier - mit einigen Weißhöhungen verwendet wird. Die etwa gleichzeitig entstandene Serie phantastischer Gebirgsszenarien (W 12396), die wankende, überkippende und explodierende Felsmassive zeigt, stellt Leonardo wiederum mit weichem Kohlestift dar. Durch die lockere Haftung des Mediums am Papiergrund partizipiert es an der Fragilität und Übergänglichkeit des Themas. Materielle Brüchigkeit und Spur, nicht feste Verfugung, bringen auch die so genannten ,Sintflutblätter' (W 12376-12386) zum Ausdruck, ein Thema, für das Leonardo um 1515 zunächst mit Feder und Lavierung experimentierte, um gleich deren Untauglichkeit festzustellen und zur schwarzen Kreide überzugehen.

Die Mimesis, besser: die Mimikry des Mediums bringt die Materialität der Darstellungsobjekte zur Anschauung. Eine ähnliche Mimikry lässt sich in der Entwicklung der Schraffurtechnik Leonardos feststellen. Als hochgradig konventionalisierte und regional erstaunlich invariante Werkstattpraxis geriet die Schraffur bisher kaum in den Blick darstellungsästhetischer Überlegungen. ${ }^{46}$ Sie war nicht Gegenstand der

45 Vgl. dazu Domenico Laurenza. „Corpus mobile. Ansätze einer Pathognomik bei Leonardo". Leonardo da Vinci. Natur im Übergang. Beiträge zu Kunst, Wissenschaft und Technik. Hg. v. Frank Fehrenbach. München, 2002, S. 257-301.

46 Vgl. Bernhard Degenhart. „Zur Graphologie der Handzeichnung. Die Strichbildung als stetige Erscheinung innerhalb der italienischen Kunstkreise". Kunstgeschichtliches Jahrbuch der Bibliotheca Hertziana 1 (1937), S. 223-343. (Ich danke Heiko Damm für diesen Hinweis.) - Auch in der jüngsten, historisch weit ausgreifenden und theoretisch ambitionierten Untersuchung der Gattung „Hand- 
literarischen Debatte; folglich existiert für eine historische Hermeneutik kein orientierendes Begriffsinstrumentarium. Das erstaunt, denn gerade an der zeichnerischen Darstellung des Dreidimensionalen werden die geläufigen Unterscheidungen von Mimesis und Modell hinfällig. Die graphischen Übersetzungen des Gegenstandes gehören ebenso sehr dem Bereich der Nachahmung wie der Konstruktion an. ${ }^{47}$ Die Strichlagen, die das Objekt zur Erscheinung bringen, verschwinden nicht hinter der gegenständlichen Illusion, sondern halten den Gegenstand gleichsam in der Schwebe des ästhetischen Scheins.

Schon der junge Leonardo experimentierte mit vielfältigen Schraffurtechniken, wofür die berühmte Landschaftszeichnung von 1473 stehen mag. ${ }^{48}$ In Mailand elaborierte Leonardo einen veränderten Stil. Es dominiert die mit äußerster Akkuratesse gezeichnete und immer enger gesetzte Parallelschraffur (Abb. 12). Dabei entsteht der Eindruck eines „dicht prasselnde[n], gleichmäßig schräge[n] Regen[s]“, wie Anny Popp 1928 schrieb $^{49}$; eines „Regens“, der zugleich den Papiergrund bezeichnet, aus dem die dargestellten Volumen zart abgestuft hervortreten. Virtuos gehandhabt, wird mit Hilfe dieser Technik das darzustellende Volumen in feinsten Schichten so abgetragen, dass rundplastische Körper hervortreten können. Im Hintergrund steht eindeutig das Vorbild des zeitgenössischen Kupferstichs. ${ }^{50}$

Dieses graphische Verfahren tendiert aber dazu, Bewegungsdarstellungen gleichsam, einzufrieren'. Nun besteht die deutlichste Veränderung in Leonardos gesamtem künstlerischem Werk darin, dass die geradlinige Diagonalschraffur durch eine kurvierte Schraffur seit etwa 1500 ergänzt und teilweise verdrängt wird. Das Verfahren setzt sich erstmals im früheren der beiden Madrider Codices in Szene, die erst 1966 wiederentdeckt wurden. Etwa 1495 entstanden, handelt es sich um das elaborierteste Manuskript Leonardos. Es ist ganz der Axiomatik

zeichnung" von David Rosand wird die Schraffur nicht systematisch behandelt (Drawing Acts. Studies in Graphic Expression and Representation. Cambridge, Mass., 2002). Überzeugende Beobachtungen zur Taktilität der Schraffurtypen: ebd., S. 107 u. 110f. (Leonardo); zur Zeitlichkeit: S. 111 (Leonardo) u. S. 206f. (Michelangelo).

47 Zum nichtmimetischen und nichtkonventionellen Charakter der zeichnerischen Urelemente vgl. Meyer Schapiro „On Some Problems in the Semiotics of Visual Art. Field and Vehicle in Image-Signs". Theory and Philosophy of Art. Style, Artist, and Society. New York, 1994, S. 1-32 (bes. S. 27f.).

48 Uffizien, GDS Inv. Nr. 8P.

49 Anny E. Popp. Leonardo da Vinci. Zeichnungen. München, 1928, S. 25.

50 Vgl. dazu Konrad Oberhuber, Jay A. Levenson u. Jacquelyn L. Sheehan. Early Italian Engravings from the National Gallery of Art. Washington, 1973, S. XVff. 


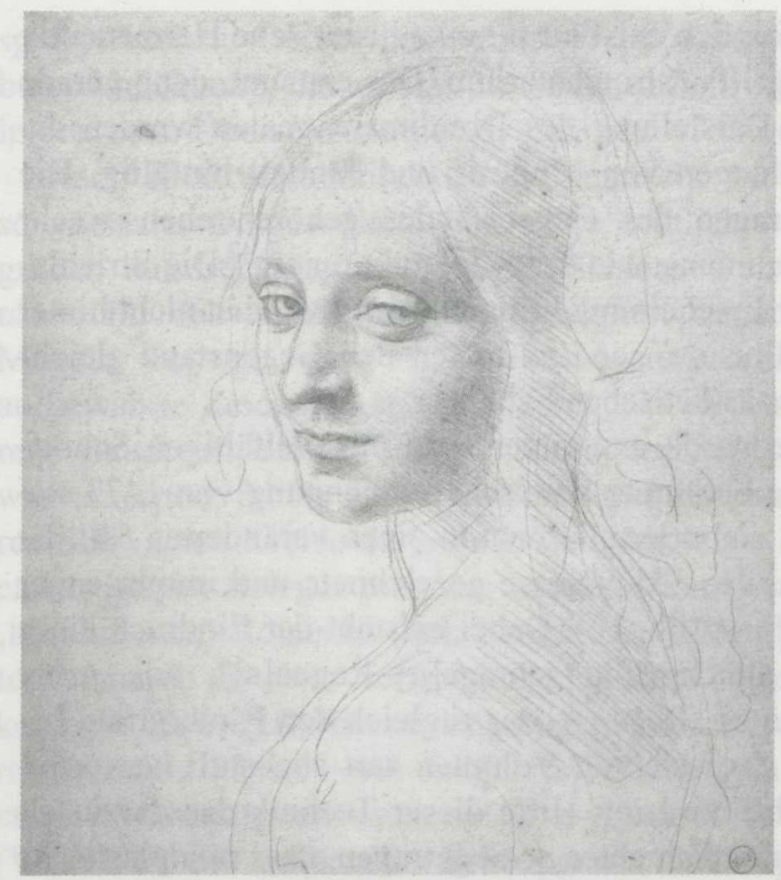

Abb. 12: Leonardo da Vinci. Kopfstudie. Silberstift, um 1485. Turin, Biblioteca Reale, Nr. 15572.

des Mechanischen gewidmet. Leonardo erörtert die theoretischen Grundlagen mechanischer Kräfte und stellt in Reihen mechanische Urelemente (Hebel, Feder, Welle etc.) vor, ein Alphabet des Mechanischen, das jeder maschinellen Applikation vorausgeht. Damit vollzieht Leonardo historisch erstmals den Schritt vom Maschinenbau zur mechanischen Grundlagenforschung, oder besser: von der Technik zur Technologie. ${ }^{51}$ Das greifen die Maschinenbücher mit gehöriger Verspätung seit dem Ende des 16. Jahrhunderts auf (z.B. bei Salomon de Caus).

Die Elemente des komponierten Maschinenkörpers werden nun in Leonardos Manuskript häufig mit kurvierter Schraffur dargestellt. ${ }^{52}$ In Codex Madrid I, fol. 45r (Abb. 13) zeichnet Leonardo beispielsweise eine Feder, die in einem Uhrwerk Verwendung finden soll. Der kurviert schraffierte Körper erscheint monumentalisiert und mit plastischer Ei-

51 Vgl. Galluzzi (Anm. 31), S. 47ff.

52 Vgl. dazu Pietro C. Marani. „Leonardo dalla scienza all'arte; un cambiamento di stile, gli antefatti, una cronologia“. Fra Rinascimento, Manierismo e Realtà. Scritti di storia dell 'arte in memoria di Anna Maria Brizio. 2. Aufl. Hg. v. dems. Florenz, 1984, S. 41-52. 


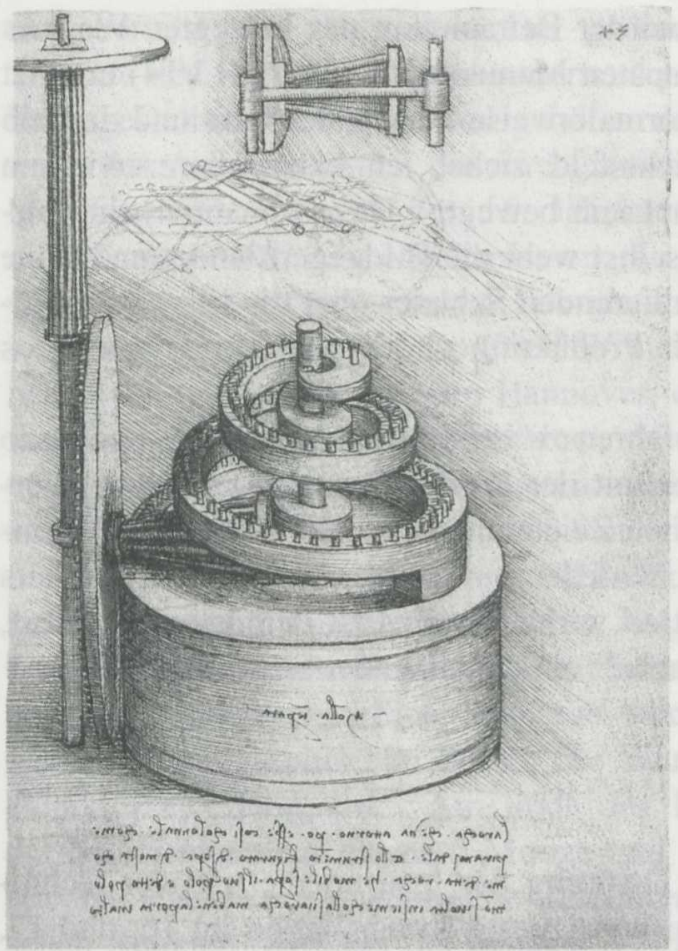

Abb. 13: Leonardo da Vinci. Federwerk. Feder, um 1492. Codex Madrid I, fol. 45r. Madrid, Biblioteca Nacional.

gendynamik ausgestattet. Seine Funktion - eine langsame kreisförmige Abgabe der gespeicherten Kraft - wird durch die Schraffur geradezu physiognomisch zum Ausdruck gebracht. Es ist, als habe Leonardo das adäquate zeichnerische Instrumentarium für Vitruvs berühmte Definition der machina entdeckt: „Machina est continens e materia coniunctio, maximas ad onerum motus habens virtutes. Ea movetur ex arte circulo-

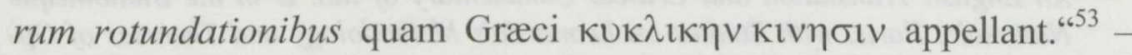
Auch im mechanischen Alphabet Leonardos sind jene wirkungsästhetischen Intentionen sichtbar, von denen bereits die Rede war: Perspektivierung, Geometrisierung, Prozessualisierung. Selbst die Einzelelemente des maschinellen Organismus erscheinen ,rhetorisiert'.

Dabei findet eine eigentümliche Inversion statt, die das aufnehmende Auge des Betrachters mit der modellierenden Hand des Künstler-Technikers kurzschließt. Schon im Manuskript A (ca. 1492) betonte

53 Vitruv. Baukunst [De architectura]. Übs. v. August Rode. Zürich u.a., 1987 [Nachdruck d. Ausgabe Leipzig, 1796], Bd. X, S. i [Herv. F. F.]. 
Leonardo, dass die Augen bei der Betrachtung des bewegten Wassers nie unbewegt bleiben. ${ }^{54}$ Im späten Manuskript E (ca. 1513/14) ergänzt Leonardo, dass das Sehen normalerweise bewegt verlaufe und deshalb eine Linie durch das Gesichtsfeld ziehe; ich extrapoliere: wie ein Zeichner, der den Punkt zur Linie bewegt. ${ }^{55}$ Davon profitiert die kurvierte Schraffur. Das Auge selbst webt die Bildgegenstände, und seine Bewegung wirft einen modellierenden Schleier über die bewegten Dinge, mit deren zeichnerischer Produktion und expansiver Dynamik es verschmilzt. $^{56}$

Auch dabei ist jenes Verfahren zu beobachten, durch das Leonardo den privilegierten Betrachter mit der Produktion der Darstellung verknüpft. Auch seine technischen Zeichnungen schließen das hervorbringende Instrument - die Zeichenfeder - mit dem Auge des Betrachters kurz. Pointiert: Die Suggestion wirkt, denn es ist der Adressat selbst, der sie erzeugt. - Jeder Betrachter ein ,zeichnender Souverän`!

Zeichnerische Rhetorik am Ursprung der Instrumente und der technischen Verfahren: Die Fortwirkung dieser Konstellation im 16. und 17. Jahrhundert wäre ein eigenes und dringendes Forschungsprojekt, das nun nicht nur die gedruckten, sondern vor allem die handschriftlichen technikgeschichtlichen Quellen breit erfassen müsste. ${ }^{57}$ So viel lässt

54, ,S]e tu riguardi il movimento dell'acqua, l'occhio tuo non si può fermare ma fa a similitudine delle cose vedute." Manuskript $A$, fol. $58 \mathrm{v}$.

55 Vgl. Manuskript E, fol. 80v; ferner 34v, 35r (im Kontext der Transformationsgeometrie vgl. Matilde Macagno. Geometry in Motion in the Manuscripts of Leonardo da Vinci. Iowa City, 1987). Zum Zusammenhang zwischen kurvierter Schraffur und bewegtem Sehen vgl. auch Donald S. Strong. Leonardo on the Eye. An English Translation and Critical Commentary of MS. D in the Bibliotheque Nationale, Paris, with Studies on Leonardo's Methodology and Theories of Optics. New York, 1979, S. 408f.

$56 \mathrm{Vgl}$. dagegen das technikgeschichtlich unfruchtbare, Gotthold Ephraim Lessing folgende Dogma bei Daniela Lamberini „It is impossible to represent movement on a two-dimensional surface (and dynamic motion is the intrinsic characteristic of the machina, as opposed to the fabrica, or building, the static machine par excellence)“. Daniela Lamberini. „Machines in Perspective. Technical Drawings in Unpublished Treatises and Notebooks of the Italian Renaissance". The Treatise on Perspective. Published and Unpublished (= Studies in the History of Art 59 Symposium Papers XXXVI). Hg. v. Lyle Massey. New Haven u. London, 2003, S. 213-233 [214].

57 Vgl. den technikgeschichtlichen Überblick von Hélène Vérin. La gloire des ingénieurs. L'intelligence technique du XVIe au XVIIle siècle. Paris, 1993. 
sich bereits jetzt feststellen: Die gedruckten ingenieurstechnischen Traktate des 16. und 17. Jahrhunderts wenden sich vorwiegend an den hoch gestellten Liebhaber und gehören damit hauptsächlich dem Bereich des lusus zu. Auch die raren und hoch geschätzten dedizierten Maschinen-Manuskripte des 16. Jahrhunderts stellen ihre (teilweise nicht funktionsfähigen) Mechanismen, die in ihren Perspektivkonstruktionen zumeist auf Francesco di Giorgio zurückgehen, häufig in makelloser Vollständigkeit dar. Gottfried Wilhelm Leibniz lehnt 1671 in einem Brief an den Herzog von Hannover, den späteren König Georg I. von England, diese ,toten“ Maschinen ab, die - unbewegt - für die distanzierte, wir können extrapolieren: ästhetische Kontemplation geschaffen wurden. ${ }^{58} \mathrm{Zu}$ dieser Zeit waren die singulären, ,physiognomischen' Fähigkeiten Leonardos, Maschinen und Maschinenelemente zu dynamisieren und zu rhetorisieren, längst der graphischen Konvention und dem immer wichtigeren dreidimensionalen Modellbau ${ }^{59}$ gewichen. Technikgeschichtlich lebte die zeichnerische Handschrift bloß noch im stenographischen Milieu der Werkstattbücher und in der Architekturzeichnung fort. ${ }^{60}$ Am Ende des 18. Jahrhunderts bereinigten der Militäringenieur Gaspard Monge und sein Mitarbeiter Jean Pierre Nicolas Hachette die technische Zeichnung von ihren letzten pathetischen Relikten (darunter der perspektivische Bezug zwischen Betrachter und funktionalem Objekt). Übrig blieb das tabellarisch geordnete Maschinenelement als Diagramm und Schnitt. ${ }^{61}$

58 Vgl. Lambertini (Anm. 56), S. 213 (im Anschluss an Manlio Brusatin. Storia delle linee. Turin, 1993, S. 52).

59 Vgl. zum Verhältnis von Zeichnung und Modell auch Massimo Scolari. „Elementi per una storia dell'assonometria“. Casabella 550 (März 1984), S. 42-49.

60 Vgl. dazu etwa Werner Oechslin. „Von Piranesi zu Libeskind. Erklären mit Zeichnung“. Daidalos 1 (1981), S. 15-19.

61 Vgl. dazu Sandrina Khaled. „Pikturale Graphismen der Technik, 1569-1870“. Bilder in Prozessen (= Bildwelten des Wissens. Kunsthistorisches Jahrbuch für Bildkritik. Bd. 1,1). Hg. v. Horst Bredekamp u. Gabriele Werner. Berlin, 2003, S. 64-78. 


\section{LITERATURVERZEICHNIS}

Alberti, Leon Battista. Das Standbild. Die Malkunst. Grundlagen der Malerei. Übs., eingel., komm. u. hg. v. Oskar Bätschmann u. Christoph Schäublin. Darmstadt, 2000.

Ames-Lewis, Francis. La matita nera nella pratica di disegno di Leonardo da Vinci (= Lettura Vinciana Bd. 41). Florenz, 2002.

Aristoteles. De partibus animalium/Parts of animals. Griech./engl. Übs. v. A. L. Peck. Cambridge, Mass., 1983.

Bacher, Jutta. „Das Theatrum machinarum. Eine Schaubühne zwischen Nutzen und Vergnügen". Erkenntnis - Erfindung - Konstruktion. Studien zur Bildgeschichte von Naturwissenschaften und Technik vom 16. bis zum 19. Jahrhundert. Hg. v. Hans Holländer. Berlin, 2000, S. 509-518.

Bacher, Jutta. ,,Ingenium vires superat'. Die Emanzipation der Mechanik und ihr Verhältnis zu Ars, Scientia und Philosophia“. Erkenntnis - Erfindung - Konstruktion. Studien zur Bildgeschichte von Naturwissenschaften und Technik vom 16. bis zum 19. Jahrhundert. Hg. v. Hans Holländer. Berlin, 2000, S. 519-555.

Barkan, Leonard. Unearthing the Past. Archeology and Aesthetics in the Making of Renaissance Culture. New Haven u. London, 1999.

Bredekamp, Horst. „Der Mensch als ,zweiter Gott‘. Motive der Wiederkehr eines kunsttheoretischen Topos im Zeitalter der Bildsimulation". Interface 1. Elektronische Medien und künstlerische Kreativität. Hg. v. Klaus Peter Dencker. Hamburg, 1992, S. 134-147.

Bredekamp, Horst. Antikensehnsucht und Maschinenglauben. Die Geschichte der Kunstkammer und die Zukunft der Kunstgeschichte. Berlin, 1993.

Britton, Pierce Dominic. „Lionizing Leonardo. A Physiognomic Conceit in Vasari's ,Vite“". Source 22.4 (2003), S. 10-15.

Brusatin, Manlio. Storia delle linee. Turin, 1993.

Butters, Suzanne B. The Triumph of Vulcan. Sculptor's Tools, Porphyry, and the Prince in Ducal Florence. 2 Bde. Florenz, 1996.

Castiglione, Baldassare. Das Buch vom Hofmann. Übs. u. hg. v. Fritz Baumgart. München, 1986.

Clark, Kenneth. The Drawings of Leonardo da Vinci in the Collection of H.M. The Queen at Windsor Castle. 3 Bde. London, 1968.

Costamagna, Philippe. Pontormo. Mailand, 1994.

Degenhart, Bernhard. „Zur Graphologie der Handzeichnung. Die Strichbildung als stetige Erscheinung innerhalb der italienischen Kunstkreise“. Kunstgeschichtliches Jahrbuch der Bibliotheca Hertziana 1 (1937), S. 223-343.

Didi-Huberman, Georges. Vor einem Bild. Übs. v. Reinold Werner. München, 2000.

Farago, Claire J. Leonardo da Vinci's ,Paragone: A Critical Interpretation with a New Edition of the Text of the ,Codex Urbinas '. Leiden u.a., 1992.

Fehrenbach, Frank. Licht und Wasser. Zur Dynamik naturphilosophischer Leitbilder im Werk Leonardo da Vincis. Tübingen, 1997.

Fehrenbach, Frank. „Hier stehe ich, aber mein Auge durcheilt die Räume. Die Vogelstudien und Flugversuche von Leonardo da Vinci“. Frankfurter Allgemeine Zeitung (27.4.2002), S. 47.

Fehrenbach, Frank. „Komposition“. Metzler Lexikon Kunstwissenschaft. Ideen, Methoden, Begriffe. Hg. v. Ulrich Pfisterer. Stuttgart u. Weimar, 2003, S. 178-183.

Furttenbach, Joseph. Mechanische Reißladen. Das ist, Ein gar geschmeidige, bey sich verborgen tragende Laden [...]. Augsburg, 1644. 
Galluzzi, Paolo (Hg.). Prima di Leonardo. Cultura delle macchine a Siena nel Rinascimento. Mailand, 1991.

Galluzzi, Paolo. Gli ingegneri del Rinascimento da Brunelleschi a Leonardo da Vinci. Florenz, 2001.

Hambly, Maja. Drawing Instruments. 1580-1980. London, 1988.

Holländer, Hans. „Spielformen der Mathesis universalis“. Erkenntnis - Erfindung Konstruktion. Studien zur Bildgeschichte von Naturwissenschaften und Technik vom 16. bis zum 19. Jahrhundert. Hg. v. dems. Berlin, 2000, S. 325-345.

Kemp, Martin. The Science of Art. Optical Themes in Western Art from Brunelleschi to Seurat. New Haven u. London, 1990.

Kemp, Wolfgang. ,... einen wahrhaft bildenden Zeichenunterricht überall einzuführen: Zeichnen und Zeichenunterricht der Laien 1500-1870. Ein Handbuch. Frankfurt a.M., 1979.

Khaled, Sandrina. „Pikturale Graphismen der Technik, 1569-1870“. Bilder in Prozessen (= Bildwelten des Wissens. Kunsthistorisches Jahrbuch für Bildkritik Bd. 1,1). Hg. v. Horst Bredekamp u. Gabriele Werner. Berlin, 2003, S. 64-78.

Körner, Hans. Auf der Suche nach der, wahren Einheit '. Ganzheitsvorstellungen in der französischen Malerei und Kunstliteratur vom mittleren 17. bis zum mittleren 19. Jahrhundert. München, 1988.

Koerner, Joseph Leo. The Moment of Self-Portraiture in German Renaissance Art. Chicago, 1993.

Krohn, Wolfgang. „Technik, Kunst und Wissenschaft. Die Idee einer konstruktiven Naturwissenschaft des Schönen bei Leon Battista Alberti“. Leonardo da Vinci. Natur im Übergang. Beiträge zu Kunst, Wissenschaft und Technik. Hg. v. Frank Fehrenbach. München, 2002, S. 37-56.

Lamberini, Daniela. „All'ombra della cupola: tradizione e innovazione nei cantieri fiorentini quattro e cinquecenteschi“. Annali di architettura 10-11 (1998/99), S. 276287.

Lamberini, Daniela. „Machines in Perspective. Technical Drawings in Unpublished Treatises and Notebooks of the Italian Renaissance". The Treatise on Perspective. Published and Unpublished (= Studies in the History of Art 59 - Symposium Papers XXXVI). Hg. v. Lyle Massey. New Haven u. London, 2003, S. 213-233.

Laurenza, Domenico. „Leonardo. Le macchine volanti“. Le macchine del Rinascimento. Hg. v. Giovanni Morello. Rom, 2000, S. 145-187.

Laurenza, Domenico. „Corpus mobile. Ansätze einer Pathognomik bei Leonardo“. Leonardo da Vinci. Natur im Übergang. Beiträge zu Kunst, Wissenschaft und Technik. Hg. v. Frank Fehrenbach. München, 2002, S. 257-301.

Leonardo da Vinci. Il Codice Atlantico di Leonardo da Vinci nella Biblioteca Ambrosiana di Milano. Hg. v. d. Accademia dei Lincei. Transkrib. v. Augusto Marinoni. 24 Bde. Florenz, 1973-80.

Leonardo da Vinci. Codices Madrid. Hg. v. Ladislao Reti u. Augusto Marinoni. 5 Bde. Frankfurt a.M., 1974.

Leonardo da Vinci. Libro di pittura. Hg. v. Carlo Pedretti. Florenz, 1995.

Leonardo da Vinci. Il codice Arundel 263 nella British Library. Hg. v. Carlo Pedretti. Transkrib. u. komm. v. Carlo Vecce. Florenz, 1998.

Macagno, Matilde. Geometry in Motion in the Manuscripts of Leonardo da Vinci. Iowa City, 1987

Marani, Pietro C. „Leonardo dalla scienza all'arte; un cambiamento di stile, gli antefatti, una cronologia“. Fra Rinascimento, Manierismo e Realtà. Scritti di storia dell'arte in memoria di Anna Maria Brizio. 2. Aufl. Hg. v. dems. Florenz, 1984, S. 41-52. 
Maschat, Herbert. Leonardo da Vinci und die Technik der Renaissance. München, 1989.

Maurice, Klaus. Der drechselnde Souverän. Materialien zu einer fürstlichen Maschinenkunst. Zürich, 1985.

Oberhuber, Konrad, Jay A. Levenson u. Jacquelyn L. Sheehan. Early Italian Engravings from the National Gallery of Art. Washington, 1973.

Oechslin, Werner. „Von Piranesi zu Libeskind. Erklären mit Zeichnung“. Daidalos 1 (1981), S. 15-19.

Ovitt, George. The Restoration of Perfection. Labor and Technology in Medieval Culture. New Brunswick u. London, 1987.

Paniagua, Cecilio. „Notes on a Drawing by Leonardo da Vinci“. International Review of Psychoanalysis 13 (1986), S. 445-452.

Pastor, Ludwig von (Hg.). Antonio de Beatis. Die Reise des Kardinals Luigi d' Aragona durch Deutschland, die Niederlande, Frankreich und Oberitalien 1517 bis 1518. Freiburg, 1905.

Pedretti, Carlo. Leonardo da Vinci on Painting. A Lost Book (Libro A). Berkeley u. Los Angeles, 1964.

Pedretti, Carlo. The Literary Works of Leonardo da Vinci. Commentary. 2 Bde. London, 1977.

Pfisterer, Ulrich (Hg.). Die Kunstliteratur der italienischen Renaissance. Eine Geschichte in Quellen. Stuttgart, 2002.

Plinius d. Ä., Gaius. Historia naturalis/Naturkunde. Lat./deutsch. Übs. u. hg. v. Roderich König. 36 Bde. München u. Darmstadt, $1973 \mathrm{ff}$.

Popp, Anny E. Leonardo da Vinci. Zeichnungen. München, 1928.

Puttfarken, Thomas. The Discovery of Pictorial Composition. Theories of Visual Order in Painting 1400-1800. New Haven u. London, 2000.

Reti, Ladislao. „Leonardo the Technologist. The Problem of the Prime Mover". Ders. u. Bern Dibner. Leonardo da Vinci, Technologist. Norwalk, 1969.

Rosand, David. Drawing Acts. Studies in Graphic Expression and Representation. Cambridge, Mass., 2002.

Rubinstein, Nicolai. „Machiavelli and the Decoration of the Hall of the Great Council in the Palazzo Vecchio“. Festschrift für Wolfram Prinz. Hg. v. Ronald G. Kecks. Berlin, 1991, S. 275-285.

Schapiro, Meyer. „On Some Problems in the Semiotics of Visual Art. Field and Vehicle in Image-Signs". Theory and Philosophy of Art. Style, Artist, and Society. New York, 1994, S. 1-32.

Scolari, Massimo. „Elementi per una storia dell'assonometria“. Casabella 550 (März 1984), S. 42-49.

Strong, Donald S. Leonardo on the Eye. An English Translation and Critical Commentary of MS. D in the Bibliothèque Nationale, Paris, with Studies on Leonardo's Methodology and Theories of Optics. New York, 1979.

Summers, David. The Judgment of Sense. Renaissance Naturalism and the Rise of Aesthetics. 2. Aufl. Cambridge, 1990.

Vasari, Giorgio. Le Vite de' più eccellenti pittori, scultori e architettori. Hg. v. Rosanna Bettarini u. Paola Barocchi. 6 Bde. Florenz, 1966-1987.

Veldman, Ilja M. u. Ger Luijten. Maarten van Heemskerck. (= The New Hollstein. Dutch \& Flemish Etchings, Engravings, and Woodcuts 1450-1700). 2 Bde. Amsterdam, 1994.

Vérin, Hélène. La gloire des ingénieurs. L'intelligence technique du XVIe au XVIIIe siècle. Paris, 1993. 
Vitruv. Baukunst. Übs. v. August Rode. 2 Bde. Zürich u.a., 1987 [Nachdruck d. Ausgabe Leipzig, 1796].

Wiemers, Michael. Bildform und Werkgenese. Studien zur zeichnerischen Bildvorbereitung in der italienischen Malerei zwischen 1450 und 1490. München u. Berlin, 1996.

Williams, Robert. Art, Theory, and Culture in Sixteenth-Century Italy. From Techne to Metatechne. Cambridge, 1997.

Woods-Marsden, Joanna. Renaissance Self-Portraiture. The Visual Construction of Identity and the Social Status of the Artist. New Haven u. London, 1998. 